\title{
Rate Analysis or a Possible Interpretation of Abundances
}

\author{
Miklós Kiss ${ }^{1}$ \\ Berze High School/Gyöngyösi Berze Nagy János Gimnázium \\ H-3200 Gyöngyös, Kossuth u. 33., Hungary \\ E-mail: kiss-mechello.hu
}

Heavy elements are formed in nucleosynthesis processes. Abundances of these elements can be classified as elemental abundance, isotopic abundance, and abundance of nuclei. In this work the nuclei are identified by $(\mathrm{Z}, \mathrm{N})$, which allows reading out new information from the measured abundances. We are interested in the neutron density required to reproduce the measured abundance of nuclei assuming equilibrium processes. This is only possible when two stable nuclei are separated by an unstable nucleus. At these places we investigated the neutron density required for equilibrium nucleosynthesis both isotopically and isotonically at temperatures of AGB interpulse and thermal pulse phases. We obtained an estimate for equilibrium nucleosynthesis neutron density in most of the cases. Next we investigated the possibility of partial formation of nuclei. We analyzed the meaning of the branching factor. We found a mathematical definition for the unified interpretation of a branching point closed at isotonic case and open at isotopic case. We introduce a more expressive variant of branching ratio called partial formation rate. With these we are capable of determining the characteristic neutron density values. We found that all experienced isotope ratios can be obtained both at $10^{8} \mathrm{~K}$ temperature and at $3 \cdot 10^{8} \mathrm{~K}$ temperature and at intermediate neutron density $\left(\leq 2 \cdot 10^{12} \mathrm{~cm}^{-3}\right)$.

XIII Nuclei in the Cosmos

7-11 July, 2014

Debrecen, Hungary

1 


\section{Introduction}

Nearly sixty years after BBFH [1], it is possible and necessary to review and rethink our knowledge about the neutron capture nucleosynthesis. The result of the formation of the nuclei is shown in the abundances. It is important to mention that the formed unstable nuclei have decayed into stable nuclei and we are only able to see the resulting stable nuclei.

"The success of any theory of nucleosynthesis has to be measured by comparison with the abundance patterns observed in nature." say Käppeler, Beer and Wisshak [2], that is, we need to create such model that gives back the observed abundance.

Because of the formation of nuclei takes place in a variety of conditions, the experienced abundance is a result of more processes. Therefore more models are necessary for the alternate conditions. According to the conditions of the models the nuclei are classified into categories as s-nuclei, r-nuclei etc.

It seems that the reverse approach is also useful: the abundance is the preserver of the nuclei's formation conditions. So instead investigating whether the theoretical model fits the observed abundance, we look for the circumstances when the observed abundance is available.

To do this we need suitable data: the half-life of unstable nuclei and the neutron capture cross section of nuclei. These data are not constant always. At some nuclei the half-lives depend on the temperature $[2,3,4]$. Fortunately, the reaction rate per particle pair $\langle\sigma v\rangle$ is constant between 10 and100 keV because of the energy dependence of $\sigma[2,3]$. So we can use the $\sigma$ values at $30 \mathrm{keV}[5]$. The possible resonances only improve the capture capabilities.

\section{The required neutron density}

Change the nucleon identification from the usual $(\mathrm{Z}, \mathrm{A})$ to $(\mathrm{Z}, \mathrm{N})$ and see the individual abundances as well. We took the abundance of nuclei from [6]. This will allow us to read new information from the various measured abundances.

We use the following rate equations according to the requirements of the individual nuclei formation:

$$
\begin{aligned}
\frac{\mathrm{dN}_{Z, N}}{\mathrm{dt}} & \left.=\mathrm{N}_{\mathrm{n}}(\mathrm{t}) \mathrm{N}_{Z, \mathrm{~N}-1}(\mathrm{t})<\sigma \mathrm{v}\right\rangle_{Z, \mathrm{~N}-1}+\lambda_{\beta} \mathrm{N}_{Z-1, \mathrm{~N}+1}(\mathrm{t})+\lambda_{\alpha} \mathrm{N}_{Z+2, \mathrm{~N}+2}(\mathrm{t})- \\
& \left.-\mathrm{N}_{\mathrm{n}}(\mathrm{t}) \mathrm{N}_{Z, \mathrm{~N}}(\mathrm{t})<\sigma \mathrm{v}\right\rangle_{Z, \mathrm{~N}}-\lambda_{\beta} \mathrm{N}_{Z, \mathrm{~N}}(\mathrm{t})-\lambda_{\alpha} \mathrm{N}_{Z, \mathrm{~N}}(\mathrm{t})+\ldots
\end{aligned}
$$

We also assume the equilibrium formation of nuclei. From the corresponding rate equations we can get the neutron density as in isotopic as in isotonic cases.

\subsection{Isotopic case}

\subsubsection{Two stable neighbor isotopes}

First let us we consider two stable neighbor isotopes (see Fig. 1), applying the new identification.

$$
\frac{\mathrm{dN}_{2}}{\mathrm{dt}}=\mathrm{n}_{\mathrm{n}} \mathrm{N}_{1}<\sigma \mathrm{v}>_{1}-\mathrm{n}_{\mathrm{n}} \mathrm{N}_{2}<\sigma \mathrm{v}>_{2}
$$




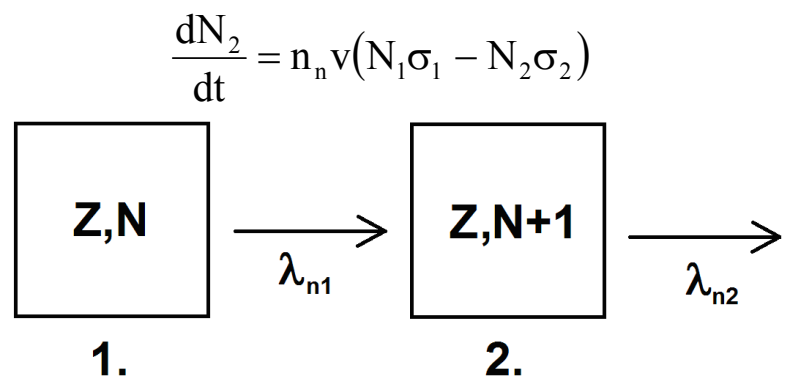

Fig. 1. Two stable neighbor isotopes

If we suppose the equilibrium that is $\frac{\mathrm{dN}_{2}}{\mathrm{dt}}=0$, we got $\mathrm{N}_{1} \sigma_{1}=\mathrm{N}_{2} \sigma_{2}$, what is well known in classical identification. So there is no information about the formation conditions. But it is important to mention that this relation is not true in general about the experienced abundances and neutron capture cross sections. The main cause is the existences of other rates from other channels through unstable nuclei.

\subsubsection{Two stable isotopes are separated by an unstable isotope}

A more interesting case is, when two stable isotopes are separated by an unstable isotope (see Fig. 2).

In this isotopic case the rate equations for the unstable nucleus:

$$
\frac{\mathrm{dN}_{2}}{\mathrm{dt}}=\mathrm{n}_{\mathrm{n}} \mathrm{N}_{1}<\sigma \mathrm{v}>_{1}-\mathrm{n}_{\mathrm{n}} \mathrm{N}_{2}<\sigma \mathrm{v}>_{2}-\lambda_{\beta} \mathrm{N}_{2}
$$

About the equilibrium $\frac{\mathrm{dN}_{2}}{\mathrm{dt}}=0$, so

$$
\begin{gathered}
0=\mathrm{n}_{\mathrm{n}}\left(\mathrm{N}_{1}<\sigma \mathrm{v}>_{1}-\mathrm{N}_{2}<\sigma \mathrm{v}>_{2}\right)-\lambda_{\beta} \mathrm{N}_{2} \\
\lambda_{\beta} \mathrm{N}_{2}=\mathrm{n}_{\mathrm{n}}\left(\mathrm{N}_{1}<\sigma \mathrm{v}>_{1}-\mathrm{N}_{2}<\sigma \mathrm{v}>_{2}\right)
\end{gathered}
$$

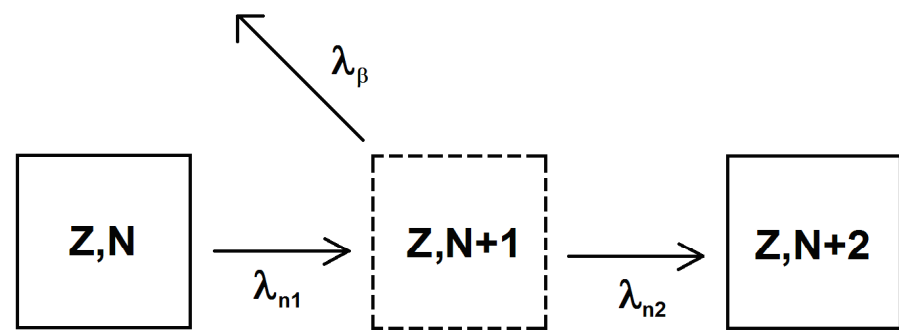

1.

2.

3.

Fig. 2. The isotopic channel

$$
\lambda_{\beta} \mathrm{N}_{2}=\mathrm{n}_{\mathrm{n}} \mathrm{v}\left(\mathrm{N}_{1} \sigma_{1}-\mathrm{N}_{2} \sigma_{2}\right)
$$




$$
\lambda_{\beta}=\mathrm{n}_{\mathrm{n}} \mathrm{v}\left(\frac{\mathrm{N}_{1}}{\mathrm{~N}_{2}} \sigma_{1}-\sigma_{2}\right)
$$

If the third nucleus can form only from the second nucleus

$$
\frac{\mathrm{dN}_{3}}{\mathrm{dt}}=\mathrm{n}_{\mathrm{n}} \mathrm{N}_{2}<\sigma \mathrm{V}>_{2}-\mathrm{n}_{\mathrm{n}} \mathrm{N}_{3}<\sigma \mathrm{V}>_{3}
$$

About the equilibrium $\frac{\mathrm{dN}_{3}}{\mathrm{dt}}=0$, so such as the previous case we have

$$
\begin{aligned}
& \mathrm{N}_{2} \sigma_{2}=\mathrm{N}_{3} \sigma_{3} \\
& \mathrm{~N}_{2}=\mathrm{N}_{3} \frac{\sigma_{3}}{\sigma_{2}} \\
& \lambda_{\beta}=\mathrm{n}_{\mathrm{n}} \mathrm{v}\left(\sigma_{1} \frac{\mathrm{N}_{1} \sigma_{2}}{\mathrm{~N}_{3} \sigma_{3}}-\sigma_{2}\right) \\
& \frac{\lambda_{\beta}}{\mathrm{n}_{\mathrm{n}} \mathrm{V}}+\sigma_{2}=\frac{\mathrm{N}_{1} \sigma_{1} \sigma_{2}}{\mathrm{~N}_{3} \sigma_{3}} \\
& \frac{\mathrm{N}_{3}}{\mathrm{~N}_{1}}=\frac{\sigma_{1} \sigma_{2}}{\left(\frac{\lambda_{\beta}}{\mathrm{n}_{\mathrm{n}} \mathrm{v}}+\sigma_{2}\right) \sigma_{3}} \\
& \frac{\mathrm{N}_{3}}{\mathrm{~N}_{1}}=\frac{\langle\sigma \mathrm{V}\rangle_{1}}{\left\langle\sigma \mathrm{v}_{3}\right\rangle} \frac{\langle\sigma \mathrm{V}\rangle_{2}}{\frac{\lambda_{\beta}}{\mathrm{n}_{\mathrm{n}}}+\langle\sigma \mathrm{V}\rangle_{2}}=\frac{\sigma_{1}}{\sigma_{3}} \frac{\sigma_{2}}{\frac{\lambda_{\beta}}{\mathrm{n}_{\mathrm{n}} \mathrm{v}}+\sigma_{2}}
\end{aligned}
$$

This expression contains the specific neutron density value. We must refer to $\langle\sigma \mathrm{v}\rangle=\sigma \cdot \mathrm{v}=$ constant at low and intermediat conditions, so it is enough to know the $30 \mathrm{keV}$ neutron capture cross sections and average velocity.

From this formula, we can get the neutron density value. Of coure resonances may occure, but these only improve the capture capabilities

$$
\mathrm{n}_{\mathrm{n}}=\frac{\lambda_{\beta}}{\mathrm{v}\left(\sigma_{1} \frac{\mathrm{N}_{1} \sigma_{2}}{\mathrm{~N}_{3} \sigma_{3}}-\sigma_{2}\right)} \text { or } \mathrm{n}_{\mathrm{n}}=\frac{\lambda_{\beta}}{\mathrm{v}} \frac{\sigma_{3}}{\sigma_{2}} \frac{1}{\left(\sigma_{1} \frac{\mathrm{N}_{1}}{\mathrm{~N}_{3}}-\sigma_{3}\right)}
$$

(See the required neutron densities at different stellar temperature in Appendix 1 and 2.)

From the tables we can see that the isotopic equilibrium is not always possible. This means that the formation of third nuclei has at least another channel.

\subsection{Isotonic case}

In this isotopic case (see Fig. 3) the rate equations for the unstable nucleus:

$$
\frac{\mathrm{dN}_{2}}{\mathrm{dt}}=\mathrm{n}_{\mathrm{n}} \mathrm{N}_{1}<\sigma \mathrm{v}>_{1}-\mathrm{n}_{\mathrm{n}} \mathrm{N}_{2}<\sigma \mathrm{v}>_{2}-\lambda_{\beta} \mathrm{N}_{2}
$$

If there is equilibrium, then $\frac{\mathrm{dN}_{2}}{\mathrm{dt}}=0$, so

$$
\mathrm{n}_{\mathrm{n}} \mathrm{N}_{1}<\sigma \mathrm{v}_{1}>=\mathrm{n}_{\mathrm{n}}<\sigma \mathrm{v}>_{2} \mathrm{~N}_{2}+\lambda_{\beta} \mathrm{N}_{2}
$$



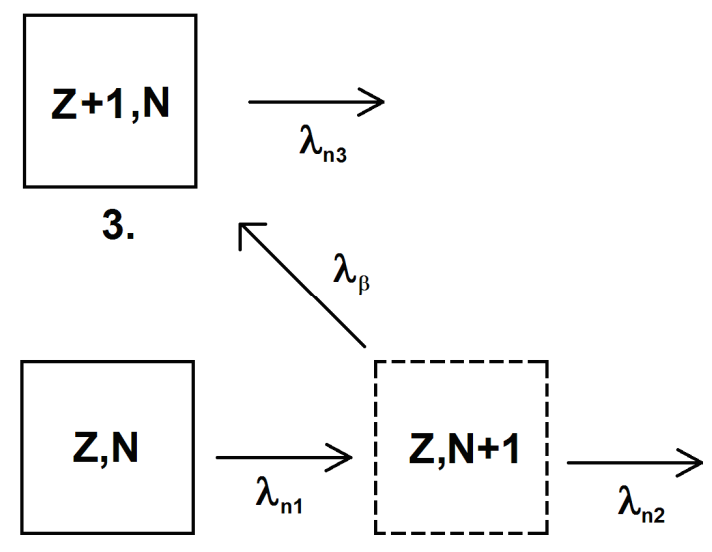

1.

2.

Fig. 3. The isotonic channel

If the third nucleus can form only from second nucleus

$$
\frac{\mathrm{dN}_{3}}{\mathrm{dt}}=\lambda_{\beta} \mathrm{N}_{2}-\mathrm{n}_{\mathrm{n}} \mathrm{N}_{3}<\sigma \mathrm{v}>_{3}
$$

Beacause of the equilibrium $\frac{\mathrm{dN}_{3}}{\mathrm{dt}}=0$ we get

The two equations

$$
\lambda_{\beta} \mathrm{N}_{2}=\mathrm{n}_{\mathrm{n}} \mathrm{N}_{3}<\sigma \mathrm{v}>_{3}
$$

about these

$$
\begin{gathered}
\mathrm{n}_{\mathrm{n}} \mathrm{N}_{1}<\sigma \mathrm{v}_{1}>=\mathrm{n}_{\mathrm{n}} \mathrm{N}_{2}<\sigma \mathrm{v}>_{2}+\lambda_{\beta} \mathrm{N}_{2} \\
\lambda_{\beta} \mathrm{N}_{2}=\mathrm{n}_{\mathrm{n}} \mathrm{N}_{3}<\sigma \mathrm{v}>_{3}
\end{gathered}
$$

$$
\begin{gathered}
\frac{\mathrm{N}_{1}}{\mathrm{~N}_{3}} \frac{<\sigma \mathrm{v}\rangle_{1}}{<\sigma>_{3}}=\frac{\left.\mathrm{n}_{\mathrm{n}}<\sigma \mathrm{v}\right\rangle_{2}+\lambda_{\beta}}{\lambda_{\beta}} \\
\frac{\mathrm{N}_{1}}{\mathrm{~N}_{3}} \frac{<\sigma \mathrm{v}>_{1}}{<\sigma \mathrm{v}>_{3}} \lambda_{\beta}-\lambda_{\beta}=\mathrm{n}_{\mathrm{n}}<\sigma \mathrm{v}>_{2}
\end{gathered}
$$

From this formula, we can get the neutron density value for equilibrium nucleosynthesis.

$$
\mathrm{n}_{\mathrm{n}}=\frac{\frac{\mathrm{N}_{1}}{\mathrm{~N}_{3}} \frac{<\sigma \mathrm{v}\rangle_{1}}{<\sigma \mathrm{v}\rangle_{3}} \lambda_{\beta}-\lambda_{\beta}}{<\sigma \mathrm{v}\rangle_{2}} \text { or } \mathrm{n}_{\mathrm{n}}=\frac{\frac{\mathrm{N}_{1}}{\mathrm{~N}_{3}} \frac{\sigma_{1}}{\sigma_{3}}-1}{\sigma_{2}} \frac{\lambda_{\beta}}{\mathrm{v}}
$$

Because of $\langle\sigma \mathrm{V}\rangle=\sigma \cdot \mathrm{v}=$ constant it is enough to take the neutron capture values at $30 \mathrm{keV}$.

(See the required neutron densities at different stellar temperature in Appendix 3 and 4.)

\subsection{The role of neutron density}

We have the possible equilibrium neutron density at isotopic and isotonic cases as well. There is a big difference between the two cases. In isotopic case the increment of neutron density increases the amount of the third nuclei, but in isotonic case the increment of neutron 
density decreases the amount of the third nuclei. These result the shift of capture path (or capture band) toward neutron rich nuclei.

\section{Partial formation of nuclei}

We can see in the previous section that not all of the third nuclei can form from the first nuclei. But how much can be formed on this way?

Suppose that from the abundance of the first nuclei $\left(\mathrm{N}_{1}\right)$ the k-times the abundance of third nuclei $\left(\mathrm{k} \cdot \mathrm{N}_{3}\right)$ are formed $(0<\mathrm{k})$. What neutron density is required in this case? (Here $\mathrm{k}$ is the Partial Formation Ratio so PFR.) If $k=1$ then all of the third nuclei are formed as in the previous section 3 .

The required neutron density at the two channels in isotopic and in isotonic cases:

$$
\mathrm{n}_{\mathrm{n}}=\frac{\lambda_{\beta}}{\mathrm{v}} \frac{\sigma_{3}}{\sigma_{2}} \frac{1}{\left(\frac{\mathrm{N}_{1} \sigma_{1}}{\mathrm{kN}_{3}}-\sigma_{3}\right)} \quad \mathrm{n}_{\mathrm{n}}=\frac{\lambda_{\beta}}{\mathrm{v}_{2}}\left(\frac{\mathrm{N}_{1}}{\mathrm{~N}_{3}} \frac{\sigma_{1}}{\sigma_{3}} \frac{1}{\mathrm{k}}-1\right)
$$

\section{Given neutron density}

After this theoretic investigation it is necessary to take a realistic approach.

We can consider the inverse question: what part of third nuclei abundance is formed from the first nuclei abundance at given neutron density? $(\mathrm{k}=$ ? $)$

\subsection{In isotopic case:}

$$
\begin{gathered}
\mathrm{k}_{\mathrm{Z}}=\mathrm{R} \frac{\lambda_{\mathrm{n}}}{\left(\lambda_{\mathrm{n}}+\lambda_{\beta}\right)}=\frac{\mathrm{N}_{1} \sigma_{1}}{\mathrm{~N}_{3} \sigma_{3}} \frac{\lambda_{\mathrm{n}}}{\left(\lambda_{\mathrm{n}}+\lambda_{\beta}\right)}=\frac{\mathrm{N}_{1} \sigma_{1}}{\mathrm{~N}_{3} \sigma_{3}} \frac{\frac{\lambda_{\mathrm{n}}}{\lambda_{\beta}}}{\left.\frac{\lambda_{\mathrm{n}}}{\lambda_{\beta}}+1\right)} \\
\mathrm{g}(\mathrm{x})_{\mathrm{Z}}=\mathrm{R} \frac{\mathrm{x}}{(\mathrm{x}+1)}=\frac{\mathrm{N}_{1} \sigma_{1}}{\mathrm{~N}_{3} \sigma_{3}} \frac{\mathrm{x}}{(\mathrm{x}+1)}=\mathrm{R} \cdot \mathrm{f}_{\mathrm{n}}(\mathrm{x}) \quad \mathrm{x}=\frac{\lambda_{\mathrm{n}}}{\lambda_{\beta}}
\end{gathered}
$$

\subsection{In isotonic case:}

$$
\begin{gathered}
\mathrm{k}_{\mathrm{N}}=\mathrm{R} \frac{\lambda_{\beta}}{\left(\lambda_{\mathrm{n}}+\lambda_{\beta}\right)}=\frac{\mathrm{N}_{1} \sigma_{1}}{\mathrm{~N}_{3} \sigma_{3}} \frac{\lambda_{\beta}}{\left(\lambda_{\mathrm{n}}+\lambda_{\beta}\right)}=\frac{\mathrm{N}_{1} \sigma_{1}}{\mathrm{~N}_{3} \sigma_{3}} \frac{1}{\left(\frac{\lambda_{\mathrm{n}}}{\lambda_{\beta}}+1\right)} \\
\mathrm{g}(\mathrm{x})_{\mathrm{N}}=\mathrm{R} \frac{1}{(\mathrm{x}+1)}=\frac{\mathrm{N}_{1} \sigma_{1}}{\mathrm{~N}_{3} \sigma_{3}} \frac{1}{(\mathrm{x}+1)}=\mathrm{R} \cdot \mathrm{f}_{\beta}(\mathrm{x}) \quad \mathrm{x}=\frac{\lambda_{\mathrm{n}}}{\lambda_{\beta}}
\end{gathered}
$$

Where $f_{n}$ and $f_{\beta}$ are the classical branching factors for neutron capture and beta decay. So we have uniform functions in both isotopic and isotonic cases. 


\section{Mathematical analysis}

It might be better to see these functions in logarithmic representation.

On Fig. 4 the two branching functions are: $\mathrm{f}_{\mathrm{n}}(\mathrm{x})$ and $\mathrm{f}_{\beta}(\mathrm{x})$, where $\xi=\lg \mathrm{x}$ :

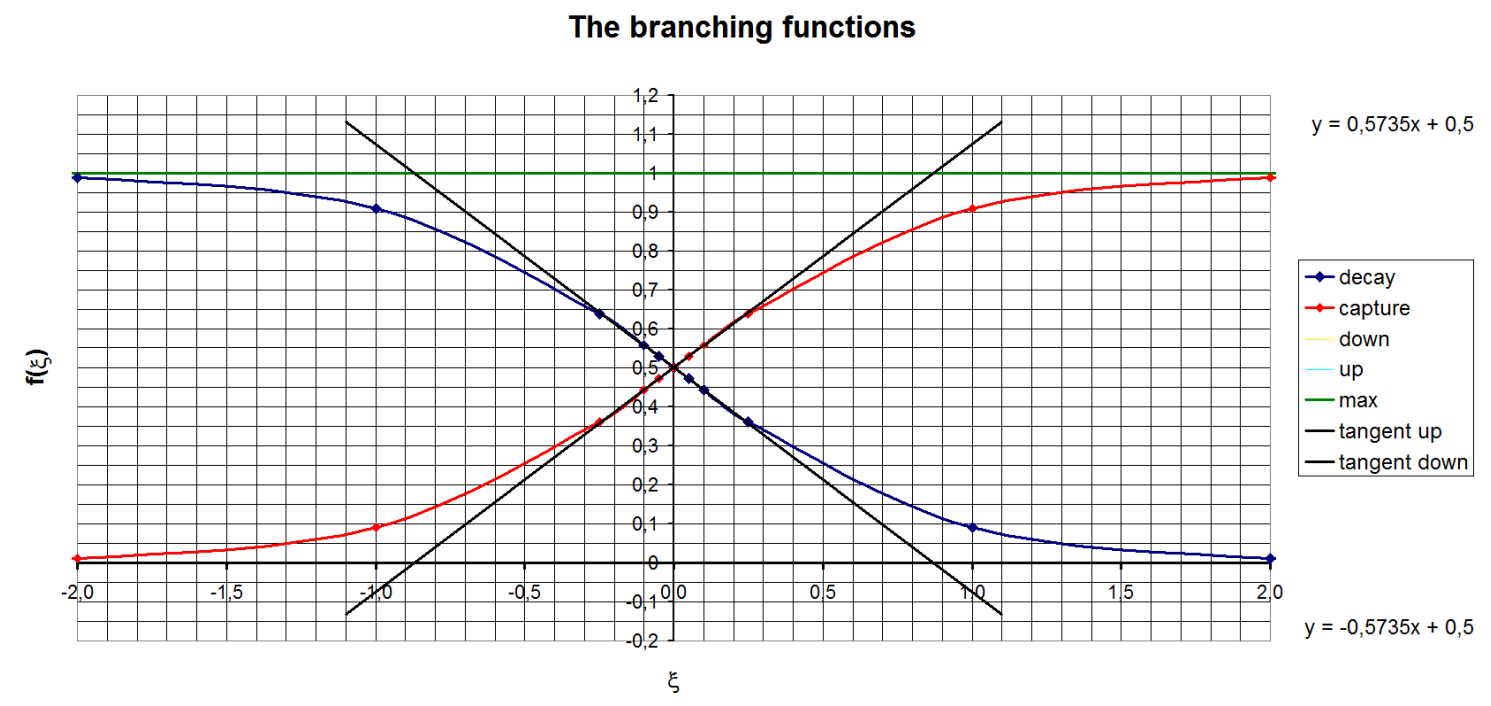

Fig. 4. The two branching functions in logharitmic scale

In this logarithmic representation the two functions become symmetric. Then we take the two logarithmic tangents at $\xi=0$ or $\mathrm{x}=1$.

Where these tangents take the value zero the isotopic formation of nuclei opens, and the isotonic formation of nuclei closes. Similarly, where the tangent takes value one, the isotopic formation is on and the isotonic formation is off. So we have got well-defined characteristics for both of the opening and closing cases.

We found a mathematical definition for the unified interpretation of a branching point closed at the isotonic case and open at the isotopic case.

\section{The neutron density range}

From the mathematical analysis we get the next values: Tab. 1 .

\begin{tabular}{|c|c|c|c|c|c|}
\hline \multirow{2}{*}{$\xi$} & \multirow{2}{*}{$\mathrm{x}$} & \multicolumn{2}{|c|}{ branching ratio } & \multicolumn{2}{|c|}{ channel } \\
\cline { 3 - 6 } & & $\mathrm{f}_{\mathrm{n}}$ & $\mathrm{f}_{\beta}$ & isotopic & isotonic \\
\hline-0.872 & 0,135 & 0.119 & 0.881 & opening & closing \\
0,872 & 7,398 & 0.881 & 0.119 & opened & closed \\
\hline
\end{tabular}

The isotopic channel is beginning to open and isotonic channel is beginning to close when $\lambda_{\mathrm{n}} \approx 0,135 \cdot \lambda_{\beta}$. Isotonic channel is nearly closed and isotopic channel is almost fully opened when $\lambda_{\mathrm{n}} \approx 7,39 \cdot \lambda_{\beta}$. We have reviewed the appropriate three nuclei cases and examined both of $\mathrm{T}_{8}$ and $3 \mathrm{~T}_{8}$ cases. 
At these cases we have got the maximum of all neutron density not more than $\mathrm{n}_{\mathrm{n}}=10^{14} \mathrm{~cm}^{-3}$. Some details are shown on the Fig. 5. Upper lines refer to isotonic cases; the bottom lines refer to isotonic cases. The temperature dependence of half-lives we took from [4].

The typical neutron density range at neutron capture nucleosynthesis

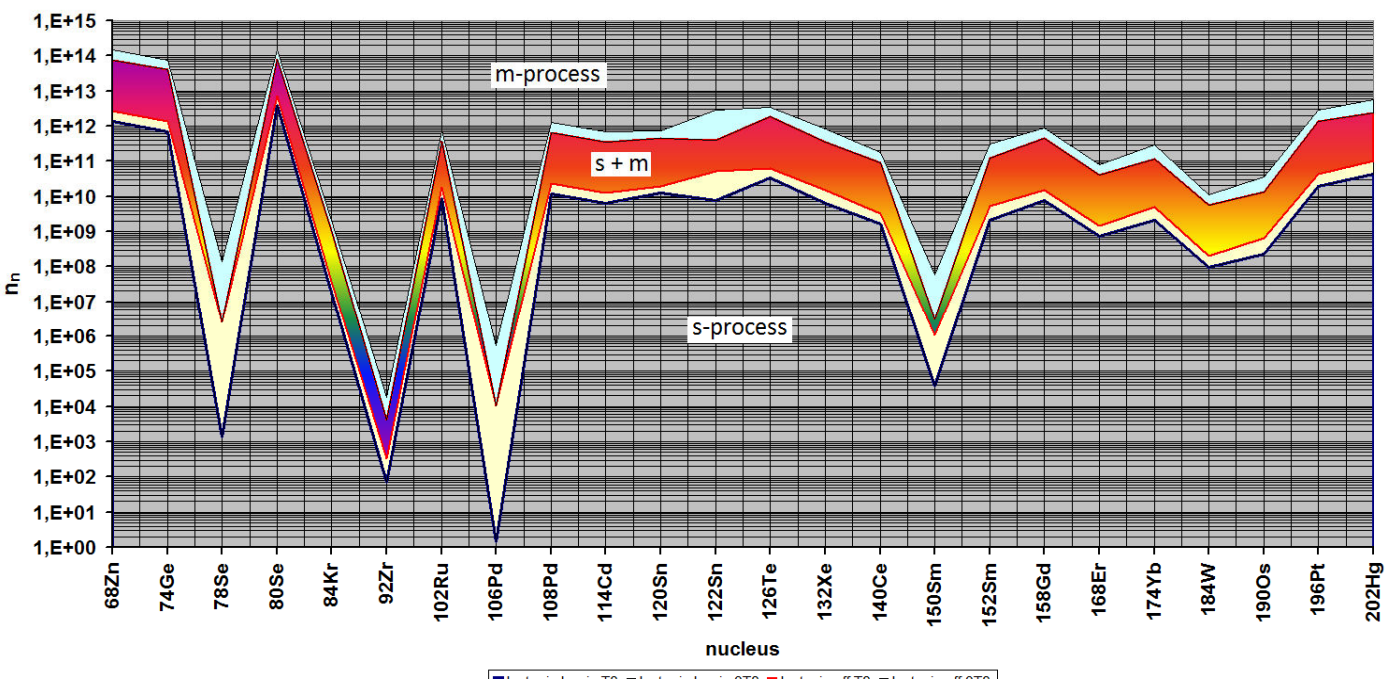

Fig. 5. The range of neutron capture nucleosynthesis about neutron density.

All nuclei formation is available at a neutron density range between s-process and $r$ process. This process is m-process (i-process) (medium or interMediate) $[7,8,9,10,11]$.

\section{Branching in a new point of view}

Ru102 isotonic closing and isotopic opening

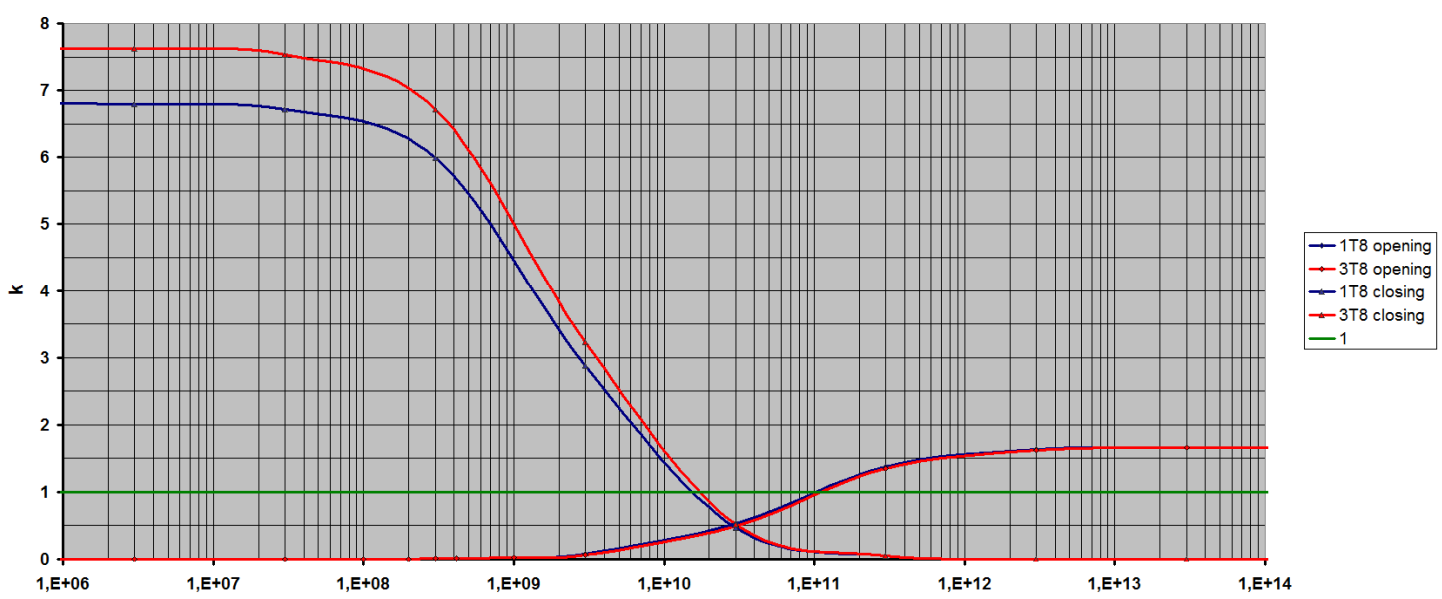

$\mathbf{n}_{\mathrm{n}}$

Fig. 6. Partial formation ratio from ${ }^{102} \mathrm{Ru}$ as the function of neutron density. Here we can the temperature dependence of the formation ratio as well. At ${ }^{102} \mathrm{Ru}$ there is no isomer.

The branching factor does not give the correct formation ratio. It gives the partial formation ratio: $k=R \cdot f$. (Data from KADoNiS [12], NNDC [13].) The $k$ is determined by $f$ 
and $R$, where $R=\frac{N_{1} \sigma_{1}}{N_{3} \sigma_{3}}$. The PFR is changing the amount of formed nuclei. The graph on Fig.

6 shows the $\mathrm{k}$ as a function of neutron density in the cases of ${ }^{102} \mathrm{Ru} \rightarrow{ }^{103} \mathrm{Rh}$ and ${ }^{102} \mathrm{Ru} \rightarrow{ }^{104} \mathrm{Ru}$ as well. These are both simple cases with only one incoming channel.

\section{Full network model}
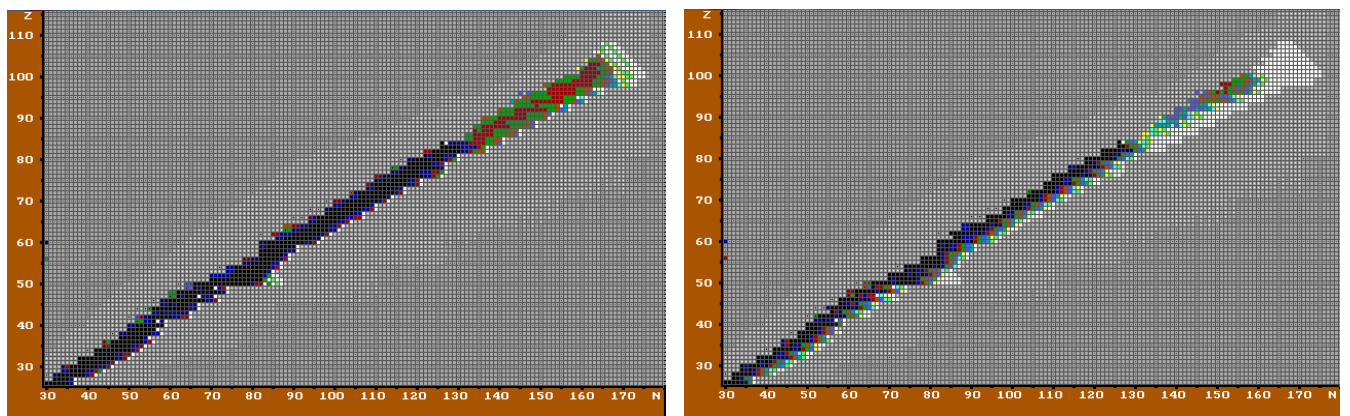

Fig. 7. The band of neutroncapture nucleosynthesis at AGP TP phase and at IP after TP

We can see in one unified model the entire possible neutron capture and decay processes $[11,14]$. Here we can change the neutron density and other parameters. But more data is required here [5]. The formation of nuclei rather occurs along a band than along a path (see Fig. 7). The neutron capture band is only visible in logarithmic representation. The structure of band at $\mathrm{Z}=50$, at tin isotopes is visible on Fig. 8 .

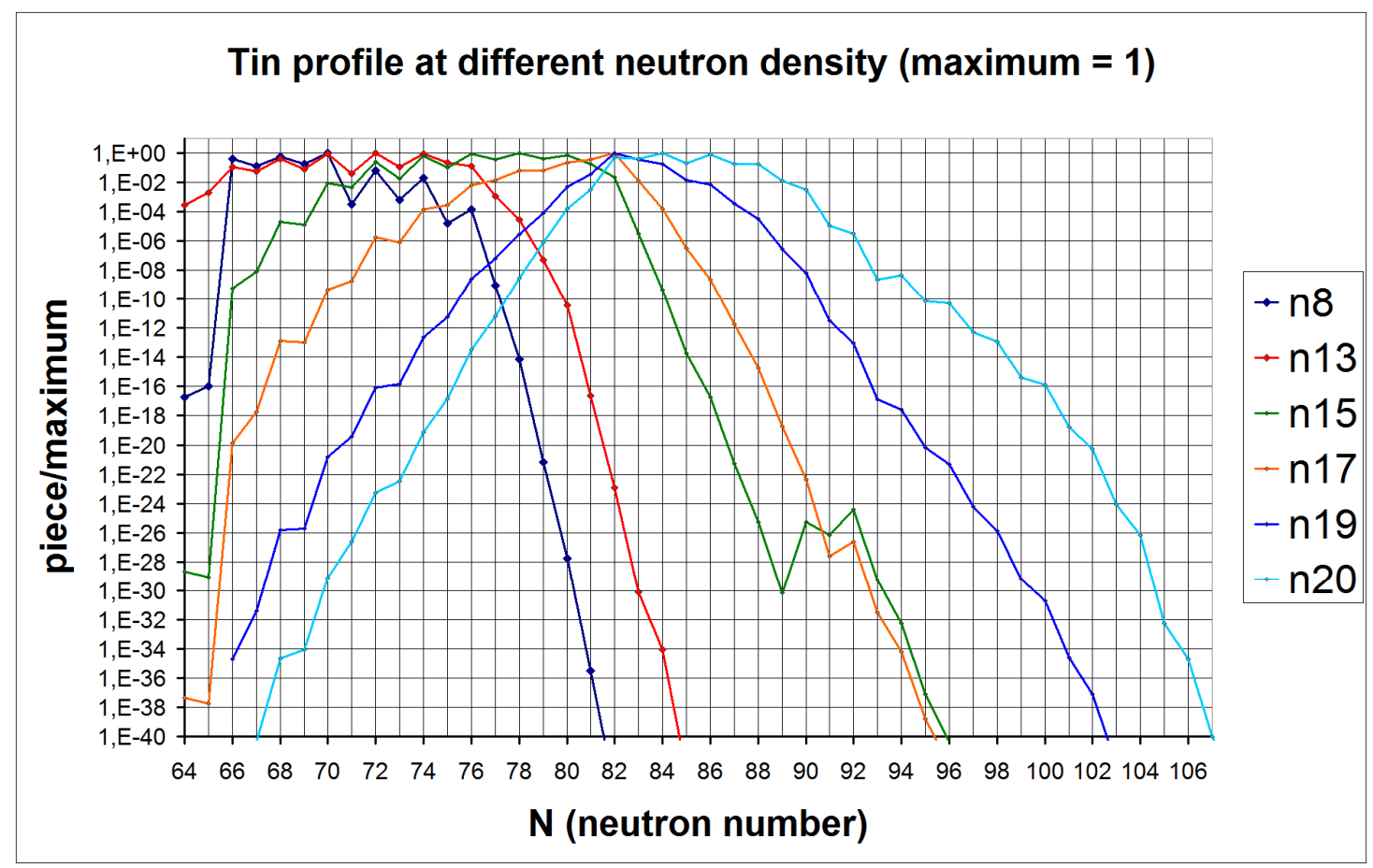

Fig. 8. The profil of neutron capture band at tin isotopes. $\left(\mathrm{n} 8=10^{8} \mathrm{~cm}^{-3}\right)$ 


\section{What does path mean at arbitrary neutron density?}

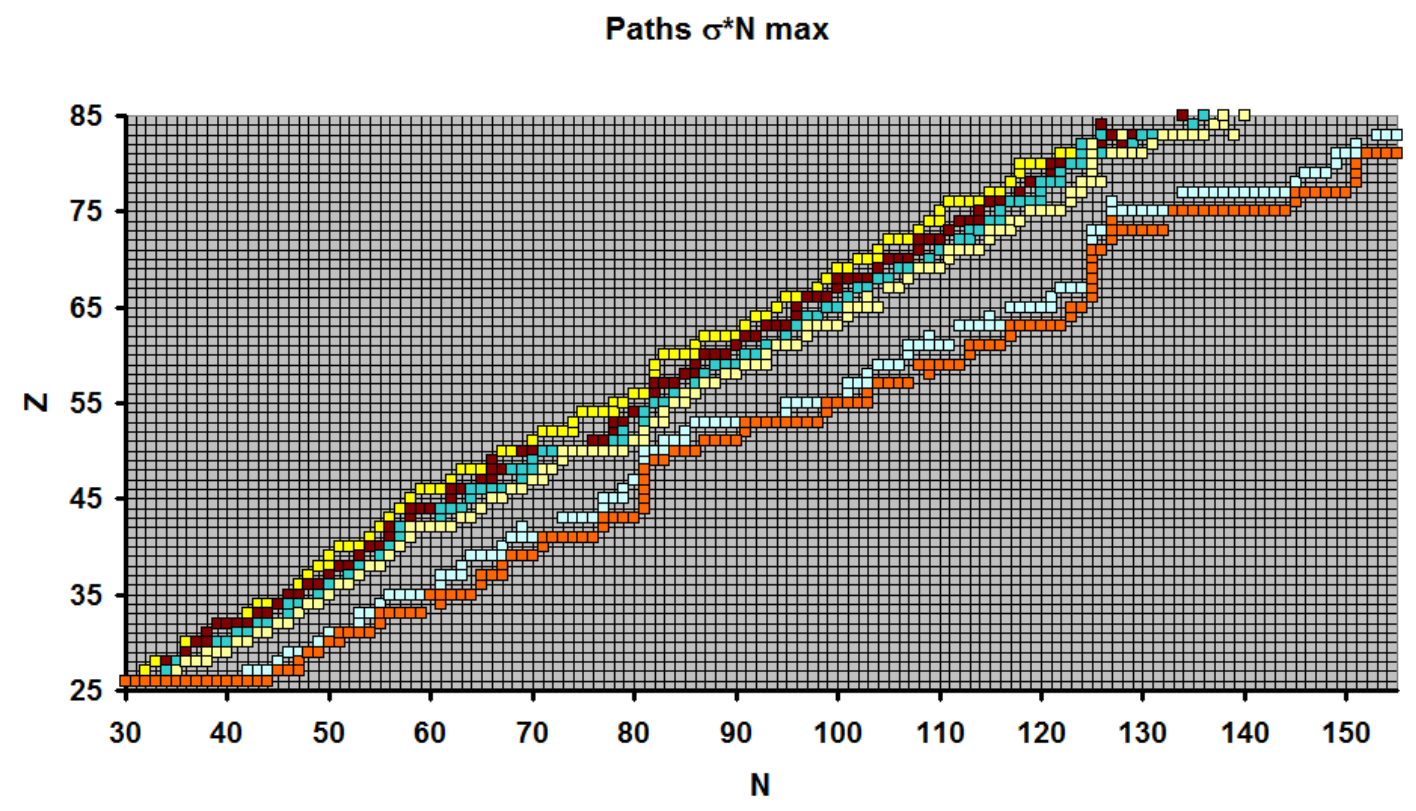

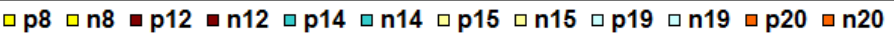

Fig. 9. The different paths at different neutron density. The maximum values we took from isotonically ( $p$ ) or isotopically $(n)$. Here $p 8$ and $n 8$ refer to the $\mathrm{n} 8=10^{8} \mathrm{~cm}^{-3}$ neutron density.

Instead of the classical paths the paths are more like the ridge of the $\sigma \mathrm{N}$ value at given neutron density, in case of the neutron capture nucleosynthesis. There is s-path, r-path and between them are the m-paths on the Fig. 9 at different neutron density by the unified model [14]. The s-path is the theoretical edge of the paths at very low neutron density.

\section{Experimental constraints: tellurium and technetium and iron}

The isotope anomalies at tellurium [11] and xenon [15] or the presence or absence of the technetium in AGB stars [16,17] can be explained with m-process. These depend on where the „path" is. We have found that the two "r-only" isotopes of tellurium $\left({ }^{128} \mathrm{Te},{ }^{130} \mathrm{Te}\right)$ are formed mainly in the m-process at AGB conditions [11].

The existence of ${ }^{60} \mathrm{Fe}$ radioisotope is also important. The stable ${ }^{58} \mathrm{Fe}$ is separated from ${ }^{60} \mathrm{Fe}$ by the unstable isotope ${ }^{59} \mathrm{Fe}$. The case is the same on the Fig. 2. The third nuclei are ${ }^{60} \mathrm{Fe}$. The rate equation for the ${ }^{59} \mathrm{Fe}$ :

$$
\begin{aligned}
& \left.\frac{\mathrm{dN}_{2}}{\mathrm{dt}}=\mathrm{n}_{\mathrm{n}} \mathrm{N}_{1}<\sigma \mathrm{v}>_{1}-\mathrm{n}_{\mathrm{n}} \mathrm{N}_{2}<\sigma \mathrm{v}\right\rangle_{2}-\lambda_{\beta 2} \mathrm{~N}_{2} \\
& \frac{\mathrm{dN}}{\mathrm{dt}}=\mathrm{n}_{\mathrm{n}} \mathrm{N}_{2}<\sigma \mathrm{v}>_{2}-\mathrm{n}_{\mathrm{n}} \mathrm{N}_{3}<\sigma \mathrm{v}>_{3}-\lambda_{\beta 3} \mathrm{~N}_{3}
\end{aligned}
$$




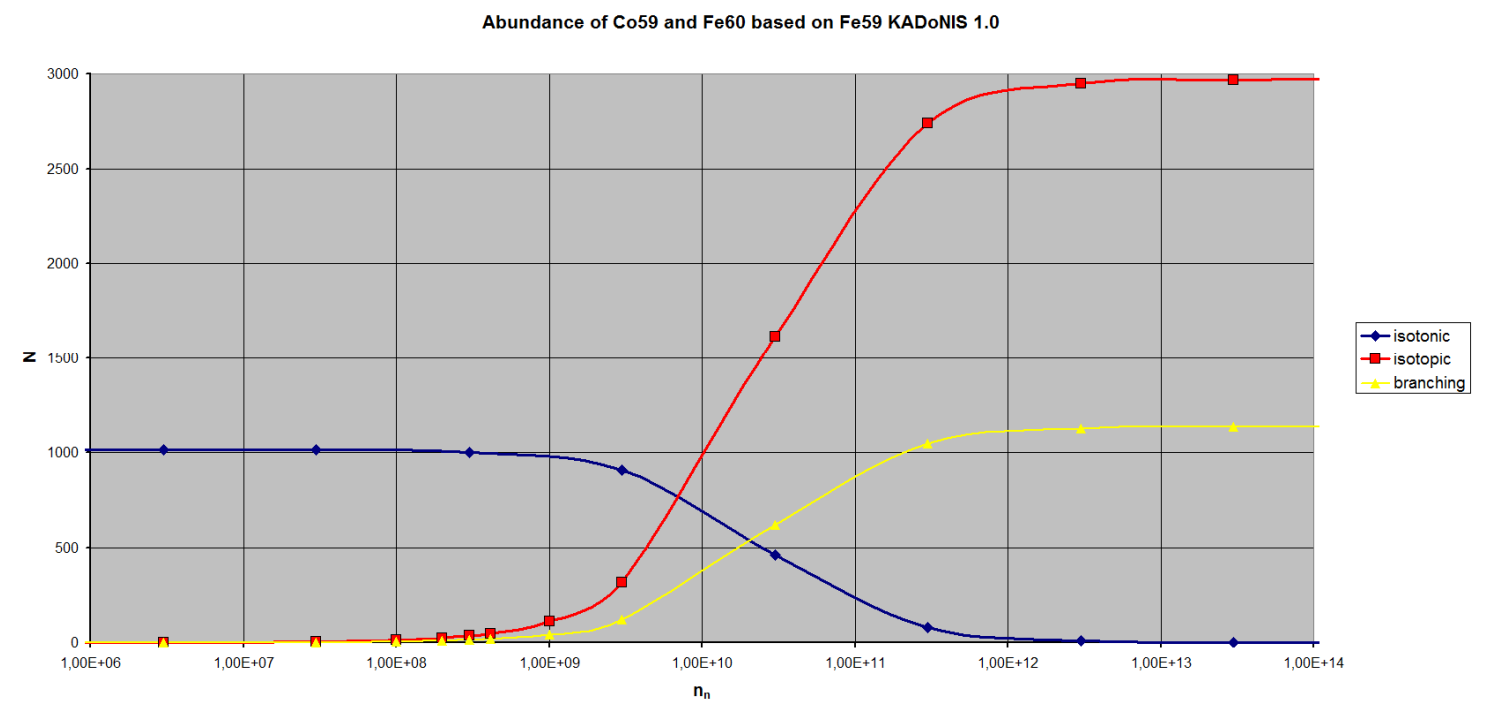

Fig. 10. The ${ }^{59} \mathrm{Co}$ and ${ }^{60} \mathrm{Fe}$ abundance at different neutrondensity. The figure shows the difference beetven PFR and the branchig factor

In the case of equilibrium comes:

$$
\mathrm{N}_{3}=\frac{\mathrm{N}_{1} \sigma_{1}}{\sigma_{3}+\frac{\lambda_{\beta 3}}{\mathrm{n}_{\mathrm{n}} \mathrm{v}}} \frac{\lambda_{\mathrm{n} 2}}{\lambda_{\mathrm{n} 2}+\lambda_{\beta 2}}=\frac{\mathrm{N}_{1} \sigma_{1}}{\sigma_{3}+\frac{\lambda_{\beta 3}}{\mathrm{n}_{\mathrm{n}} \mathrm{v}}} \mathrm{f}_{\mathrm{n} 2} \quad \mathrm{~N}_{3}=\frac{\mathrm{N}_{1} \sigma_{1}}{\sigma_{3}+\frac{\lambda_{\beta 3}}{\mathrm{n}_{\mathrm{n}} \mathrm{v}}} \frac{\mathrm{x}_{2}}{1+\mathrm{x}_{2}}
$$

Because of the half-life of ${ }^{60} \mathrm{Fe}\left(\mathrm{T}=1,5 \cdot 10^{6} \mathrm{y}\right) \frac{\lambda_{\beta 3}}{\mathrm{n}_{\mathrm{n}} \mathrm{V}}<<\sigma_{3}$, so it is negligible [18,19].

$$
\mathrm{N}_{3}=\frac{\mathrm{N}_{1} \sigma_{1}}{\sigma_{3}+\frac{\lambda_{\beta 3}}{\mathrm{n}_{\mathrm{n}} \mathrm{v}}} \frac{\mathrm{x}_{2}}{1+\mathrm{x}_{2}} \cong \frac{\mathrm{N}_{1} \sigma_{1}}{\sigma_{3}} \frac{\mathrm{x}_{2}}{1+\mathrm{x}_{2}}=\frac{\mathrm{N}_{1} \sigma_{1}}{\sigma_{3}} \mathrm{f}_{\mathrm{n} 2}=\mathrm{N}_{1} \frac{\sigma_{1}}{\sigma_{3}} \mathrm{f}_{\mathrm{n} 2}=\mathrm{N}_{1} \frac{13,5}{5,15} \mathrm{f}_{\mathrm{n} 2}=\mathrm{N}_{1} \cdot 2,62 \cdot \mathrm{f}_{\mathrm{n} 2}
$$

At a given neutron density the amount of ${ }^{60} \mathrm{Fe}$ is nearly three times what we get from the simple branching ratio (see Fig. 10). We used data from KADoNiS 1.0 [19].

This explains the high abundance of ${ }^{60} \mathrm{Ni}$, which is four times (4.34 times) the amount of ${ }^{59} \mathrm{Co}$. The formation of ${ }^{60} \mathrm{Ni}$ nuclei occurs mainly through the ${ }^{60} \mathrm{Fe}$ channel.

\section{Conclusion}

All experienced isotope ratios can be obtained both at $10^{8} \mathrm{~K}$ temperature and at $3 \cdot 10^{8} \mathrm{~K}$ temperature at intermediate neutron density $\left(10^{12}-10^{14} \mathrm{~cm}^{-3}\right)$, so the m-process and the AGB stars are probably one of the main places of nucleosynthesis. It seems that the so-called r-nuclei can form in intermediate processes as well.

\section{References}

[1] E. M. Burbidge, G. R. Burbidge, W. A. Fowler, and F. Hoyle (1957). "Synthesis of the Elements in Stars". Reviews of Modern Physics 29 (4): 547. Bibcode:1957RvMP...29..547B.

doi:10.1103/RevModPhys.29.547. 
[2] F. Käppeler, H. Beer and K. Wisshak, s-process nucleosynthesis-nuclear physics and the classical model: Rep. Prog. Phys. 52 (1989) 945-1013.

[3] C. E. Rolfs, W. S. Rodney: Cauldrons in the Cosmos, The Univ. of Chicago Press, 1988

[4] K. Takahashi, K. Yokoi: BETA-DECAY RATES OF HIGHLY IONIZED HEAVY ATOMS IN STELLAR INTERIORS, ATOMIC DATA AND NUCLEAR DATA TABLES 36,375-409 (1987)]

[5] M. Kiss and Z. Trócsányi, "Phenomenological Description of Neutron Capture Cross Sections at 30 keV,” ISRN Astronomy and Astrophysics, vol. 2013, Article ID 170954, 8 pages, 2013. doi:10.1155/2013/170954

[6] D Arnett: Supernovae and Nucleosynthesis, Princeton University Press, 1996

[7] J. J. Cowan and W. K. Rose: PRODUCTION OF ${ }^{14} C$ AND NEUTRONS IN RED GIANTS, The Astrophysical Journal, 212:149-158, 1977 February 15

[8] R. A. Malaney, Heavy -element synthesis in AGB and post-AGB stars of low mass, Mon. Not. R. astr. Soc. (1986) 223, 709-725

[9] M. Lugaro, A. I. Karakas Sara Bisterzo Models and observations of the s process in AGB, PoS(NIC X)034, 2008:

[10] P. Prado, L. Dardalet, E. Heringer, C. Higgs, C. Ritter, S. Jones, M. Pignatari, M. Bertolli, P. Woodward, Falk Herwig, i process and CEMP-s+r stars NIC XIII. 2014

[11] M. Kiss, PhD Thesis/Egyetemi doktori (PhD) értekezés, Debreceni Egyetem Debrecen 2012

[12] http://www.kadonis.org/

[13] http://www.nndc.bnl.gov/astro/

[14] Kiss M., Trócsányi Z. A unified model for nucleosynthesis of heavy elements in stars, Journal of Physics: Conference Series (2010) 012024 doi:10.1088/1742-6596/202/1/012024

[15] J. D. Gilmour and G. Turner, CONSTRAINTS ON NUCLEOSYNTHESIS FROM XENON ISOTOPES IN PRESOLAR MATERIAL, The Astrophysical Journal, 657:600Y608, 2007 March 1

[16] T. Lebzelter, J. Hron, Technetium and the third dredge up in AGB stars I. Field stars, A\&A 411, 533-542 (2003) doi: 10.1051/0004-6361:20031458

[17] R. A. MALANEY: Production of technetium in red giants by $\gamma$-ray-induced fission, Nature 337, 718 - 720 (23 February 1989); doi:10.1038/337718a0

[18] http://www.nndc.bnl.gov/astro/calcmacs.jsp

[19] Kadonis 1.0: http://exp-astro.physik.uni-frankfurt.de/kadonis1.0/ 


\section{Appendix}

\section{Isotopic equilibrium neutron density at a temperature of $10^{8} \mathrm{~K}$}

\begin{tabular}{|c|c|c|c|c|c|c|c|c|c|c|c|c|}
\hline & nuc $_{1}$ & $\begin{array}{l}\log y_{1} \\
(S i=6)\end{array}$ & $\sigma_{1}(\mathrm{mb})$ & nuc $_{2}$ & $\mathbf{T}_{2}$ & $\begin{array}{l}\text { time } \\
\text { unit }\end{array}$ & $\sigma_{2}(\mathrm{mb})$ & nuc $_{3}$ & $\sigma_{3}(\mathrm{mb})$ & $\begin{array}{l}\log y_{3} \\
(S i=6)\end{array}$ & $\mathrm{n}_{\mathrm{n}}\left(\mathrm{cm}^{-3}\right)$ & $\lg n_{n}$ \\
\hline 1. & $62 \mathrm{Ni}$ & 3,255 & 22,2 & $63 \mathrm{Ni}$ & 100,1 & $Y$ & 66,7 & $64 \mathrm{Ni}$ & 8,4 & 2,726 & $2,2 \mathrm{E}+06$ & 6,33 \\
\hline 2. & $68 Z n$ & 2,369 & 20,7 & $69 Z n$ & 56,4 & $\mathrm{~m}$ & $\underline{75,4}$ & $70 \mathrm{Zn}$ & 10,9 & 0,893 & $1,8 \mathrm{E}+11$ & 11,25 \\
\hline 3. & $68 \mathrm{Zn}$ & 2,369 & 20,7 & $69 \mathrm{Zn}^{\mathrm{m}}$ & 13,76 & $d^{*}$ & 110,9 & $70 \mathrm{Zn}$ & 10,9 & 0,893 & $3,5 \mathrm{E}+08$ & 8,54 \\
\hline 4. & $69 \mathrm{Ga}$ & 1,358 & 118,7 & $70 \mathrm{Ga}$ & 21,14 & $d$ & $\underline{301,5}$ & $71 \mathrm{Ga}$ & 104,6 & 1,176 & $6,4 \mathrm{E}+09$ & 9,81 \\
\hline 5. & $74 \mathrm{Ge}$ & 1,638 & 37,6 & $75 \mathrm{Ge}$ & 82,78 & $\mathrm{~m}$ & 203,2 & $76 \mathrm{Ge}$ & 24,4 & 0,965 & $4,0 \mathrm{E}+11$ & 11,61 \\
\hline 6. & $78 \mathrm{Se}$ & 1,164 & 61,1 & $79 S e$ & $2,95 \mathrm{E}+05$ & $y$ & 263 & $80 \mathrm{Se}$ & 38 & 1,49 & $-4,3 E+04$ & $\mathrm{n} / \mathrm{a}$ \\
\hline 7. & $80 \mathrm{Se}$ & 1,49 & 38 & $81 \mathrm{Se}$ & 18,45 & $\mathrm{~m}$ & $\underline{82,3}$ & $82 \mathrm{Se}$ & 8,4 & 0,757 & $1,2 \mathrm{E}+12$ & 12,08 \\
\hline 8. & $80 \mathrm{Se}$ & 1,49 & 38 & $81 \mathrm{Se}^{\mathrm{m}}$ & 57,28 & $\mathrm{~m}^{*}$ & $\underline{82,3}$ & $82 \mathrm{Se}$ & 8,4 & 0,757 & $3,8 \mathrm{E}+11$ & 11,59 \\
\hline 9. & $79 \mathrm{Br}$ & 0,775 & 622 & $80 \mathrm{Br}$ & $1,77 \mathrm{E}+01$ & $\mathrm{~m}$ & $\underline{790,0}$ & $81 \mathrm{Br}$ & 239 & 0,766 & $1,8 \mathrm{E}+12$ & 12,25 \\
\hline 10. & $79 \mathrm{Br}$ & 0,775 & 622 & $80 \mathrm{Br}^{\mathrm{m}}$ & $4,42 \mathrm{E}+00$ & $\mathrm{~h}^{*}$ & $\underline{790,0}$ & $81 \mathrm{Br}$ & 239 & 0,766 & $1,2 \mathrm{E}+11$ & 11,08 \\
\hline 11. & $84 \mathrm{Kr}$ & 1,408 & 32,6 & $85 \mathrm{Kr}$ & 3916,8 & D & 73 & $86 \mathrm{Kr}$ & 4,76 & 0,893 & $4,8 \mathrm{E}+06$ & 6,68 \\
\hline 12. & $84 \mathrm{Kr}$ & 1,408 & 32,6 & $85 \mathrm{Kr}^{\mathrm{m}}$ & 4,48 & h $78,6 \%$ & 73 & $86 \mathrm{Kr}$ & 4,76 & 0,893 & $1,0 \mathrm{E}+11$ & 11,01 \\
\hline 13. & $85 \mathrm{Rb}$ & 0,709 & 234 & $86 \mathrm{Rb}$ & 18,642 & $d$ & 202 & $87 \mathrm{Rb}$ & 15,7 & 0,316 & $2,2 \mathrm{E}+08$ & 8,34 \\
\hline 14. & $92 \mathrm{Zr}$ & 0,209 & 37,8 & $93 \mathrm{Zr}$ & $1,53 E+06$ & Y & 96 & $94 Z r$ & 27,3 & 0,209 & $1,3 \mathrm{E}+03$ & 3,12 \\
\hline 15. & $94 Z r$ & 0,297 & 27,3 & $95 Z r$ & 64,032 & D & 79 & $96 Z r$ & 10,3 & $-0,496$ & $3,8 \mathrm{E}+08$ & 8,58 \\
\hline 16. & $98 \mathrm{Mo}$ & $-0,218$ & 70,5 & 99Mo & 2,7489 & D & 240 & $100 \mathrm{Mo}$ & 80,5 & $-0,609$ & $3,9 \mathrm{E}+10$ & 10,59 \\
\hline 17. & $102 R u$ & $-0,231$ & 151 & $103 \mathrm{Ru}$ & 29,26 & D & 343 & $104 R u$ & 154 & $-0,461$ & $9,5 \mathrm{E}+10$ & 10,98 \\
\hline 18. & $106 \mathrm{Pd}$ & $-0,42$ & 244 & $107 \mathrm{Pd}$ & $6,50 \mathrm{E}+06$ & Y & 1302 & $108 \mathrm{Pd}$ & 218 & $-0,431$ & $7,4 \mathrm{E}+01$ & 1,87 \\
\hline 19. & $108 \mathrm{Pd}$ & $-0,431$ & 218 & $109 \mathrm{Pd}$ & 13,70012 & $\mathrm{H}$ & 236,3 & $110 P d$ & 157 & $-0,785$ & $1,0 \mathrm{E}+11$ & 11,01 \\
\hline 20. & $107 \mathrm{Ag}$ & $-0,602$ & 787 & $108 \mathrm{Ag}$ & 2,37 & $\mathrm{~m}$ & $\underline{1788,0}$ & $109 \mathrm{Ag}$ & 793 & $-0,627$ & $2,0 \mathrm{E}+14$ & 14,29 \\
\hline 21. & $107 \mathrm{Ag}$ & $-0,602$ & 787 & $108 \mathrm{Ag}^{\mathrm{m}}$ & 438 & $y^{*} e c$ & 1383,0 & $109 \mathrm{Ag}$ & 793 & $-0,627$ & $2,6 \mathrm{E}+06$ & 6,42 \\
\hline 22. & $114 \mathrm{Cd}$ & $-0,333$ & 135,3 & $115 \mathrm{Cd}$ & 53,46 & $\mathrm{H}$ & 290 & $116 C d$ & 76,1 & $-0,914$ & $7,9 \mathrm{E}+09$ & 9,90 \\
\hline 23. & $114 \mathrm{Cd}$ & $-0,333$ & 135,3 & $115 \mathrm{Cd}^{\mathrm{m}}$ & 44,56 & d & 224 & $116 C d$ & 76,1 & $-0,914$ & $5,1 \mathrm{E}+08$ & 8,71 \\
\hline 24. & $113 \mathrm{In}$ & $-2,103$ & 809 & 114 In & 71,9 & $\mathrm{~s}$ & $\underline{1308,0}$ & $115 \ln$ & 776 & $-0,754$ & $-2,8 \mathrm{E}+13$ & $\mathrm{n} / \mathrm{a}$ \\
\hline 25. & $113 \ln$ & $-2,103$ & 809 & $114 \mathrm{In}^{\mathrm{m}}$ & 49,51 & $d^{*}$ & 2595 & $115 \ln$ & 776 & $-0,754$ & $-2,4 \mathrm{E}+08$ & $\mathrm{n} / \mathrm{a}$ \\
\hline 26. & $120 S n$ & 0,097 & 36,3 & $121 S n$ & 27,03 & $\mathrm{H}$ & 167 & $122 S n$ & 22,6 & $-0,745$ & $9,1 \mathrm{E}+09$ & 9,96 \\
\hline 27. & $120 \mathrm{Sn}$ & 0,097 & 36,3 & $121 \mathrm{Sn}^{\mathrm{m}}$ & 43,9 & y $22 \%$ & 175,4 & $122 S n$ & 22,6 & $-0,745$ & $6,1 \mathrm{E}+05$ & 5,79 \\
\hline 28. & $122 S n$ & $-0,745$ & 22,6 & $123 S n$ & 129,2 & D & 361 & $124 S n$ & 15,7 & $-0,644$ & $3,9 \mathrm{E}+11$ & 11,59 \\
\hline 29. & $122 \mathrm{Sn}$ & $-0,745$ & 22,6 & $123 \mathrm{Sn}^{\mathrm{m}}$ & 40,06 & $\mathrm{~m}$ & 361 & $124 S n$ & 15,7 & $-0,644$ & $1,8 \mathrm{E}+15$ & 15,26 \\
\hline 30. & $121 \mathrm{Sb}$ & $-0,752$ & 532 & $122 \mathrm{Sb}$ & 3 & D & 894 & $123 \mathrm{Sb}$ & 303 & $-0,879$ & $9,0 \mathrm{E}+09$ & 9,95 \\
\hline 31. & $126 \mathrm{Te}$ & $-0,046$ & 81,3 & $127 \mathrm{Te}$ & 9,35 & $\mathrm{H}$ & 256,8 & $128 \mathrm{Te}$ & 44,4 & 0,185 & $3,9 \mathrm{E}+12$ & 12,59 \\
\hline 32. & $126 \mathrm{Te}$ & $-0,046$ & 81,3 & $127 \mathrm{Te}^{\mathrm{m}}$ & 109 & d $2,4 \%$ & 668,6 & $128 \mathrm{Te}$ & 44,4 & 0,185 & $1,1 \mathrm{E}+11$ & 11,05 \\
\hline 33. & $128 \mathrm{Te}$ & 0,185 & 44,4 & 129Te & 69,6 & M & $\underline{137,5}$ & $130 \mathrm{Te}$ & 14,2 & 0,22 & $2,4 \mathrm{E}+12$ & 12,37 \\
\hline
\end{tabular}




\begin{tabular}{|c|c|c|c|c|c|c|c|c|c|c|c|c|}
\hline & nuc $_{1}$ & $\begin{array}{l}\log y_{1} \\
(S i=6)\end{array}$ & $\sigma_{1}(\mathrm{mb})$ & nuc $_{2}$ & $T_{2}$ & $\begin{array}{l}\text { time } \\
\text { unit }\end{array}$ & $\sigma_{2}(\mathrm{mb})$ & $\mathrm{nuc}_{3}$ & $\sigma_{3}(\mathrm{mb})$ & $\begin{array}{l}\log y_{3} \\
(S i=6)\end{array}$ & $\mathrm{n}_{\mathrm{n}}\left(\mathrm{cm}^{-3}\right)$ & $\lg n_{n}$ \\
\hline 34. & $128 \mathrm{Te}$ & 0,185 & 44,4 & $129 \mathrm{Te}^{\mathrm{m}}$ & 33,6 & $\mathrm{~d}^{*}$ & 493,6 & $130 \mathrm{Te}$ & 14,2 & 0,22 & $9,5 \mathrm{E}+08$ & 8,98 \\
\hline 35. & $132 X e$ & 0,086 & 63,8 & $133 X e$ & 5,243 & D & 127 & $134 X e$ & 21,3 & $-0,321$ & $7,2 \mathrm{E}+09$ & 9,86 \\
\hline 36. & $134 \mathrm{Xe}$ & $-0,321$ & 21,3 & $135 X e$ & 9,14 & $\mathrm{H}$ & 65,6 & $136 \times e$ & 0,98 & $-0,405$ & $4,7 E+10$ & 10,67 \\
\hline 37. & $140 \mathrm{Ce}$ & 0,004 & 11,73 & $141 \mathrm{Ce}$ & 32,508 & D & 76 & $142 \mathrm{Ce}$ & 29,9 & $-0,9$ & $5,6 \mathrm{E}+09$ & 9,75 \\
\hline 38. & $146 \mathrm{Nd}$ & $-0,845$ & 91,2 & $147 \mathrm{Nd}$ & 10,98 & D & 544 & $148 \mathrm{Nd}$ & 146,6 & $-1,324$ & $5,7 \mathrm{E}+09$ & 9,75 \\
\hline 39. & $148 \mathrm{Nd}$ & $-1,324$ & 146,6 & $149 \mathrm{Nd}$ & 17,28 & $\mathrm{H}$ & 513,2 & $150 \mathrm{Nd}$ & 156,1 & $-1,333$ & $-1,9 E+12$ & $\mathrm{n} / \mathrm{a}$ \\
\hline 40. & $150 \mathrm{Sm}$ & $-1,717$ & 422,3 & $151 \mathrm{Sm}$ & 90 & $Y$ & 3040 & $152 \mathrm{Sm}$ & 464,8 & $-1,161$ & $-3,7 E+05$ & $\mathrm{n} / \mathrm{a}$ \\
\hline 41. & $152 \mathrm{Sm}$ & $-1,161$ & 464,8 & $153 \mathrm{Sm}$ & 46,284 & $\mathrm{H}$ & 1095 & $154 S m$ & 216,9 & $-1,232$ & $1,0 \mathrm{E}+10$ & 10,01 \\
\hline 42. & $158 \mathrm{Gd}$ & $-1,086$ & 323,6 & $159 \mathrm{Gd}$ & 18,479 & $\mathrm{H}$ & 455,2 & $160 \mathrm{Gd}$ & 178 & $-1,141$ & $7,6 \mathrm{E}+10$ & 10,88 \\
\hline 43. & $168 \mathrm{Er}$ & $-1,168$ & 319 & $169 \mathrm{Er}$ & 9,392 & D & 653,0 & $170 E r$ & 170,2 & $-1,428$ & $2,2 \mathrm{E}+09$ & 9,35 \\
\hline 44. & $174 \mathrm{Yb}$ & $-1,103$ & 150,5 & $175 \mathrm{Yb}$ & 4,185 & D & 558 & $176 \mathrm{Yb}$ & 115,9 & $-1,5$ & $6,7 \mathrm{E}+09$ & 9,83 \\
\hline 45. & $184 \mathrm{~W}$ & $-1,389$ & 225 & $185 W$ & 75,1 & D & 633 & $186 W$ & 226 & $-1,423$ & $8,8 \mathrm{E}+09$ & 9,94 \\
\hline 46. & $185 \mathrm{Re}$ & $-1,717$ & 1438,5 & 186Re & 3,7186 & $\mathrm{D}$ & 743 & $187 \operatorname{Re}$ & 1184 & $-1,475$ & $-3,5 E+10$ & $\mathrm{n} / \mathrm{a}$ \\
\hline 47. & $185 \mathrm{Re}$ & $-1,717$ & 1438,5 & $186 \operatorname{Re}^{m}$ & $2,00 E+05$ & $y^{*}$ & 743 & $187 \operatorname{Re}$ & 1184 & $-1,475$ & $-1,8 E+03$ & $n / a$ \\
\hline 48. & $1900 \mathrm{~s}$ & $-0,75$ & 278 & 1910s & 15,4 & D & 1290 & $1920 s$ & 160 & $-0,558$ & $1,4 \mathrm{E}+10$ & 10,16 \\
\hline 49. & $191 / \mathrm{r}$ & $-0,607$ & 1350 & $192 \mathrm{Ir}$ & 73,827 & D & 2080 & 193Ir & 994 & $-0,383$ & $-1,0 E+09$ & $\mathrm{n} / \mathrm{a}$ \\
\hline 50. & $191 / r$ & $-0,607$ & 1350 & $192 \mathrm{lr}^{\mathrm{m}}$ & 241 & $y^{*} e c$ & 2080 & $193 / r$ & 994 & $-0,383$ & $-8,6 \mathrm{E}+05$ & $n / a$ \\
\hline 51. & $196 \mathrm{Pt}$ & $-0,47$ & 167,4 & 197Pt & 19,8915 & $\mathrm{H}$ & 79,7 & $198 P t$ & 94 & $-1,015$ & $8,6 \mathrm{E}+10$ & 10,93 \\
\hline 52. & $202 \mathrm{Hg}$ & $-0,996$ & 63,3 & $203 \mathrm{Hg}$ & 46,594 & D & 98 & $204 \mathrm{Hg}$ & 42 & $-1,633$ & $5,7 \mathrm{E}+10$ & 10,76 \\
\hline 53. & $203 \mathrm{TI}$ & $-1,265$ & 170,5 & $204 \mathrm{TI}$ & 3,78 & $y$ & 215 & $205 \mathrm{TI}$ & 53 & $-0,866$ & $3,4 \mathrm{E}+08$ & 8,53 \\
\hline
\end{tabular}

\section{Isotopic equilibrium neutron density at a temperature of $3 \cdot 10^{8} \mathrm{~K}$}

\begin{tabular}{|c|c|c|c|c|c|c|c|c|c|c|c|c|}
\hline & nuc $_{1}$ & $\begin{array}{l}\log y_{1} \\
(S i=6)\end{array}$ & $\sigma_{1}(\mathrm{mb})$ & nuc $_{2}$ & $T_{2}$ & $\begin{array}{l}\text { time } \\
\text { unit }\end{array}$ & $\sigma_{2}(m b)$ & nuc $_{3}$ & $\sigma_{3}(\mathrm{mb})$ & $\begin{array}{l}\log y_{3} \\
(S i=6)\end{array}$ & $\mathrm{n}_{\mathrm{n}}\left(\mathrm{cm}^{-3}\right)$ & $\lg n_{n}$ \\
\hline 1. & $62 \mathrm{Ni}$ & 3,255 & 22,2 & $63 \mathrm{Ni}$ & 100,1 & $Y$ & 66,7 & $64 \mathrm{Ni}$ & 8,4 & 2,726 & $1,2 \mathrm{E}+07$ & 7,09 \\
\hline 2. & $68 Z n$ & 2,369 & 20,7 & $69 Z n$ & 56,4 & $\mathrm{~m}$ & 75,4 & $70 \mathrm{Zn}$ & 10,9 & 0,893 & $1,8 \mathrm{E}+11$ & 11,25 \\
\hline 3. & $68 Z n$ & 2,369 & 20,7 & $69 \mathrm{Zn}^{\mathrm{m}}$ & 13,76 & $d^{*}$ & 110,9 & $70 \mathrm{Zn}$ & 10,9 & 0,893 & $3,5 \mathrm{E}+08$ & 8,54 \\
\hline 4. & $69 \mathrm{Ga}$ & 1,358 & 118,7 & $70 \mathrm{Ga}$ & 21,14 & $d$ & $\underline{301,5}$ & $71 \mathrm{Ga}$ & 104,6 & 1,176 & $6,4 \mathrm{E}+09$ & 9,81 \\
\hline 5. & $74 \mathrm{Ge}$ & 1,638 & 37,6 & $75 \mathrm{Ge}$ & 82,78 & $\mathrm{~m}$ & 203,2 & $76 \mathrm{Ge}$ & 24,4 & 0,965 & $4,0 \mathrm{E}+11$ & 11,60 \\
\hline 6. & $78 \mathrm{Se}$ & 1,164 & 61,1 & $79 S e$ & $2,95 \mathrm{E}+05$ & $y$ & 263 & $80 \mathrm{Se}$ & 38 & 1,49 & $-7,6 \mathrm{E}+07$ & $\mathrm{n} / \mathrm{a}$ \\
\hline 7. & $80 \mathrm{Se}$ & 1,49 & 38 & $81 \mathrm{Se}$ & 18,45 & $\mathrm{~m}$ & $\underline{82,3}$ & $82 \mathrm{Se}$ & 8,4 & 0,757 & $1,1 \mathrm{E}+12$ & 12,05 \\
\hline 8. & $80 \mathrm{Se}$ & 1,49 & 38 & $81 \mathrm{Se}^{\mathrm{m}}$ & 57,28 & $\mathrm{~m}^{*}$ & 82,3 & $82 \mathrm{Se}$ & 8,4 & 0,757 & $3,6 \mathrm{E}+11$ & 11,55 \\
\hline 9. & $79 \mathrm{Br}$ & 0,775 & 622 & $80 \mathrm{Br}$ & $1,77 \mathrm{E}+01$ & $\mathrm{~m}$ & $\underline{790,0}$ & $81 \mathrm{Br}$ & 239 & 0,766 & $1,2 \mathrm{E}+12$ & 12,08 \\
\hline 10. & $79 \mathrm{Br}$ & 0,775 & 622 & $80 \mathrm{Br}^{\mathrm{m}}$ & $4,42 \mathrm{E}+00$ & $h^{*}$ & $\underline{790,0}$ & $81 \mathrm{Br}$ & 239 & 0,766 & $7,9 \mathrm{E}+10$ & 10,90 \\
\hline 11. & $84 \mathrm{Kr}$ & 1,408 & 32,6 & $85 \mathrm{Kr}$ & 3916,8 & D & 73 & $86 \mathrm{Kr}$ & 4,76 & 0,893 & $4,9 \mathrm{E}+06$ & 6,69 \\
\hline
\end{tabular}




\begin{tabular}{|c|c|c|c|c|c|c|c|c|c|c|c|c|}
\hline & nuc $_{1}$ & $\begin{array}{l}\log y_{1} \\
(S i=6)\end{array}$ & $\sigma_{1}(\mathrm{mb})$ & nuc $_{2}$ & $T_{2}$ & $\begin{array}{l}\text { time } \\
\text { unit }\end{array}$ & $\sigma_{2}(\mathrm{mb})$ & nuc $_{3}$ & $\sigma_{3}(\mathrm{mb})$ & $\begin{array}{l}\log y_{3} \\
(S i=6)\end{array}$ & $\mathrm{n}_{\mathrm{n}}\left(\mathrm{cm}^{-3}\right)$ & $\lg n_{n}$ \\
\hline 12. & $84 \mathrm{Kr}$ & 1,408 & 32,6 & $85 \mathrm{Kr}^{\mathrm{m}}$ & 4,48 & h $78,6 \%$ & 73 & $86 \mathrm{Kr}$ & 4,76 & 0,893 & $1,0 \mathrm{E}+11$ & 11,02 \\
\hline 13. & $85 \mathrm{Rb}$ & 0,709 & 234 & $86 \mathrm{Rb}$ & 18,642 & d & 202 & $87 \mathrm{Rb}$ & 15,7 & 0,316 & $2,3 E+11$ & 11,36 \\
\hline 14. & $92 Z r$ & 0,209 & 37,8 & $93 Z r$ & $1,53 \mathrm{E}+06$ & $Y$ & 96 & $94 Z r$ & 27,3 & 0,209 & $4,8 E+03$ & 3,68 \\
\hline 15. & $94 Z r$ & 0,297 & 27,3 & $95 \mathrm{Zr}$ & 64,032 & D & 79 & $96 Z r$ & 10,3 & $-0,496$ & $3,8 \mathrm{E}+08$ & 8,58 \\
\hline 16. & 98Mo & $-0,218$ & 70,5 & 99Mo & 2,7489 & D & 240 & $100 \mathrm{Mo}$ & 80,5 & $-0,609$ & $5,8 \mathrm{E}+10$ & 10,76 \\
\hline 17. & $102 \mathrm{Ru}$ & $-0,231$ & 151 & $103 R u$ & 29,26 & D & 343 & $104 R u$ & 154 & $-0,461$ & $1,1 \mathrm{E}+11$ & 11,03 \\
\hline 18. & $106 \mathrm{Pd}$ & $-0,42$ & 244 & $107 \mathrm{Pd}$ & $6,50 \mathrm{E}+06$ & $Y$ & 1302 & $108 \mathrm{Pd}$ & 218 & $-0,431$ & $5,2 E+05$ & 5,72 \\
\hline 19. & $108 \mathrm{Pd}$ & $-0,431$ & 218 & 109Pd & 13,70012 & $\mathrm{H}$ & 236,3 & $110 P d$ & 157 & $-0,785$ & $1,0 \mathrm{E}+11$ & 11,01 \\
\hline 20. & $107 \mathrm{Ag}$ & $-0,602$ & 787 & $108 \mathrm{Ag}$ & 2,37 & $\mathrm{~m}$ & $\underline{1788,0}$ & $109 \mathrm{Ag}$ & 793 & $-0,627$ & $1,7 \mathrm{E}+14$ & 14,23 \\
\hline 21. & $107 \mathrm{Ag}$ & $-0,602$ & 787 & $108 \mathrm{Ag}^{\mathrm{m}}$ & 438 & $y^{*} e c$ & 1383,0 & $109 \mathrm{Ag}$ & 793 & $-0,627$ & $2,3 E+06$ & 6,36 \\
\hline 22. & $114 \mathrm{Cd}$ & $-0,333$ & 135,3 & $115 \mathrm{Cd}$ & 53,46 & $\mathrm{H}$ & 290 & $116 C d$ & 76,1 & $-0,914$ & $7,9 \mathrm{E}+09$ & 9,90 \\
\hline 23. & $114 \mathrm{Cd}$ & $-0,333$ & 135,3 & $115 \mathrm{Cd}^{\mathrm{m}}$ & 44,56 & $d$ & 224 & $116 C d$ & 76,1 & $-0,914$ & $5,1 \mathrm{E}+08$ & 8,71 \\
\hline 24. & $113 \ln$ & $-2,103$ & 809 & $114 \operatorname{In}$ & 71,9 & $\mathrm{~s}$ & $\underline{1308,0}$ & $115 \ln$ & 776 & $-0,754$ & $-2,8 E+13$ & $\mathrm{n} / \mathrm{a}$ \\
\hline 25. & $113 \ln$ & $-2,103$ & 809 & $114 \ln ^{\mathrm{m}}$ & 49,51 & $d^{*}$ & 2595 & $115 \ln$ & 776 & $-0,754$ & $-2,4 \mathrm{E}+08$ & $\mathrm{n} / \mathrm{a}$ \\
\hline 26. & $120 \mathrm{Sn}$ & 0,097 & 36,3 & $121 \mathrm{Sn}$ & 27,03 & $\mathrm{H}$ & 167 & $122 S n$ & 22,6 & $-0,745$ & $4,6 \mathrm{E}+09$ & 9,66 \\
\hline 27. & $120 \mathrm{Sn}$ & 0,097 & 36,3 & $121 \mathrm{Sn}^{\mathrm{m}}$ & 43,9 & y $22 \%$ & 175,4 & $122 S n$ & 22,6 & $-0,745$ & $3,0 E+05$ & 5,48 \\
\hline 28. & $122 \mathrm{Sn}$ & $-0,745$ & 22,6 & $123 \mathrm{Sn}$ & 129,2 & D & 361 & $124 S n$ & 15,7 & $-0,644$ & $2,4 \mathrm{E}+12$ & 12,38 \\
\hline 29. & $122 \mathrm{Sn}$ & $-0,745$ & 22,6 & $123 \mathrm{Sn}^{\mathrm{m}}$ & 40,06 & $\mathrm{~m}$ & 361 & $124 S n$ & 15,7 & $-0,644$ & $1,1 \mathrm{E}+16$ & 16,04 \\
\hline 30. & $121 \mathrm{Sb}$ & $-0,752$ & 532 & $122 \mathrm{Sb}$ & 3 & D & 894 & $123 \mathrm{Sb}$ & 303 & $-0,879$ & $3,4 \mathrm{E}+10$ & 10,53 \\
\hline 31. & $126 \mathrm{Te}$ & $-0,046$ & 81,3 & $127 \mathrm{Te}$ & 9,35 & $\mathrm{H}$ & 256,8 & $128 T e$ & 44,4 & 0,185 & $3,4 \mathrm{E}+12$ & 12,53 \\
\hline 32. & $126 \mathrm{Te}$ & $-0,046$ & 81,3 & $127 \mathrm{Te}^{\mathrm{m}}$ & 109 & d $2,4 \%$ & 668,6 & $128 T e$ & 44,4 & 0,185 & $9,6 \mathrm{E}+10$ & 10,98 \\
\hline 33. & $128 \mathrm{Te}$ & 0,185 & 44,4 & $129 \mathrm{Te}$ & 69,6 & M & $\underline{137,5}$ & $130 \mathrm{Te}$ & 14,2 & 0,22 & $2,2 \mathrm{E}+12$ & 12,35 \\
\hline 34. & $128 \mathrm{Te}$ & 0,185 & 44,4 & $129 \mathrm{Te}^{\mathrm{m}}$ & 33,6 & $d^{*}$ & 493,6 & $130 \mathrm{Te}$ & 14,2 & 0,22 & $9,0 \mathrm{E}+08$ & 8,95 \\
\hline 35. & $132 X e$ & 0,086 & 63,8 & $133 X e$ & 5,243 & D & 127 & $134 X e$ & 21,3 & $-0,321$ & $9,4 \mathrm{E}+09$ & 9,97 \\
\hline 36. & $134 \mathrm{Xe}$ & $-0,321$ & 21,3 & $135 \mathrm{Xe}$ & 9,14 & $\mathrm{H}$ & 65,6 & $136 \times e$ & 0,98 & $-0,405$ & $4,7 \mathrm{E}+10$ & 10,67 \\
\hline 37. & $140 \mathrm{Ce}$ & 0,004 & 11,73 & $141 \mathrm{Ce}$ & 32,508 & D & 76 & $142 \mathrm{Ce}$ & 29,9 & $-0,9$ & $5,6 \mathrm{E}+09$ & 9,75 \\
\hline 38. & $146 \mathrm{Nd}$ & $-0,845$ & 91,2 & $147 \mathrm{Nd}$ & 10,98 & $\mathrm{D}$ & 544 & $148 \mathrm{Nd}$ & 146,6 & $-1,324$ & $5,7 \mathrm{E}+09$ & 9,75 \\
\hline 39. & $148 \mathrm{Nd}$ & $-1,324$ & 146,6 & $149 \mathrm{Nd}$ & 17,28 & $\mathrm{H}$ & 513,2 & $150 \mathrm{Nd}$ & 156,1 & $-1,333$ & $-1,9 \mathrm{E}+12$ & $n / a$ \\
\hline 40. & $150 \mathrm{Sm}$ & $-1,717$ & 422,3 & $151 \mathrm{Sm}$ & 90 & Y & 3040 & $152 \mathrm{Sm}$ & 464,8 & $-1,161$ & $-9,8 E+06$ & $n / a$ \\
\hline 41. & $152 \mathrm{Sm}$ & $-1,161$ & 464,8 & $153 \mathrm{Sm}$ & 46,284 & $\mathrm{H}$ & 1095 & $154 S m$ & 216,9 & $-1,232$ & $1,6 \mathrm{E}+10$ & 10,20 \\
\hline 42. & $158 \mathrm{Gd}$ & $-1,086$ & 323,6 & $159 \mathrm{Gd}$ & 18,479 & $\mathrm{H}$ & 455,2 & $160 \mathrm{Gd}$ & 178 & $-1,141$ & $7,6 \mathrm{E}+10$ & 10,88 \\
\hline 43. & $168 \mathrm{Er}$ & $-1,168$ & 319 & $169 \mathrm{Er}$ & 9,392 & D & 653,0 & $170 E r$ & 170,2 & $-1,428$ & $2,2 \mathrm{E}+09$ & 9,33 \\
\hline 44. & $174 \mathrm{Yb}$ & $-1,103$ & 150,5 & $175 \mathrm{Yb}$ & 4,185 & D & 558 & $176 \mathrm{Yb}$ & 115,9 & $-1,5$ & $1,0 \mathrm{E}+10$ & 10,00 \\
\hline 45. & $184 \mathrm{~W}$ & $-1,389$ & 225 & $185 W$ & 75,1 & D & 633 & $186 W$ & 226 & $-1,423$ & $9,5 \mathrm{E}+09$ & 9,98 \\
\hline 46. & $185 \mathrm{Re}$ & $-1,717$ & 1438,5 & $186 \operatorname{Re}$ & 3,7186 & $\mathrm{D}$ & 743 & $187 \mathrm{Re}$ & 1184 & $-1,475$ & $-3,8 E+10$ & $\mathrm{n} / \mathrm{a}$ \\
\hline 47. & $185 \mathrm{Re}$ & $-1,717$ & 1438,5 & $186 \mathrm{Re}^{\mathrm{m}}$ & $2,00 \mathrm{E}+05$ & $\mathrm{y}^{*} \mathrm{ec}$ & 743 & $187 \mathrm{Re}$ & 1184 & $-1,475$ & $-1,9 \mathrm{E}+03$ & $\mathrm{n} / \mathrm{a}$ \\
\hline 48. & $1900 s$ & $-0,75$ & 278 & 1910s & 15,4 & D & 1290 & $1920 s$ & 160 & $-0,558$ & $2,6 \mathrm{E}+10$ & 10,41 \\
\hline
\end{tabular}




\begin{tabular}{|c|c|c|c|c|c|c|c|c|c|c|c|c|}
\hline & nuc $_{1}$ & $\begin{array}{l}\log y_{1} \\
(S i=6)\end{array}$ & $\sigma_{1}(m b)$ & nuc $_{2}$ & $T_{2}$ & $\begin{array}{l}\text { time } \\
\text { unit }\end{array}$ & $\sigma_{2}(\mathrm{mb})$ & nuc $_{3}$ & $\sigma_{3}(\mathrm{mb})$ & $\begin{array}{l}\log y_{3} \\
(S i=6)\end{array}$ & $\mathrm{n}_{\mathrm{n}}\left(\mathrm{cm}^{-3}\right)$ & $\lg n_{n}$ \\
\hline 49. & $191 / \mathrm{r}$ & $-0,607$ & 1350 & $192 \mathrm{Ir}$ & 73,827 & D & 2080 & $193 \mid r$ & 994 & $-0,383$ & $-1,4 \mathrm{E}+09$ & $\mathrm{n} / \mathrm{a}$ \\
\hline 50. & $191 / r$ & $-0,607$ & 1350 & $192 \mathrm{Ir}^{\mathrm{m}}$ & $2,41 \mathrm{E}+02$ & $y^{*}$ & 2080 & $193 \mid r$ & 994 & $-0,383$ & $-1,2 \mathrm{E}+06$ & $\mathrm{n} / \mathrm{a}$ \\
\hline 51. & $196 \mathrm{Pt}$ & $-0,47$ & 167,4 & $197 \mathrm{Pt}$ & 19,8915 & $\mathrm{H}$ & 79,7 & $198 P t$ & 94 & $-1,015$ & $9,9 E+10$ & 11,00 \\
\hline 52. & $202 \mathrm{Hg}$ & $-0,996$ & 63,3 & $203 \mathrm{Hg}$ & 46,594 & D & 98 & $204 \mathrm{Hg}$ & 42 & $-1,633$ & $8,1 \mathrm{E}+10$ & 10,91 \\
\hline 53. & $203 T I$ & $-1,265$ & 170,5 & $204 T I$ & 3,78 & $y$ & 215 & $205 \mathrm{TI}$ & 53 & $-0,866$ & $6,8 \mathrm{E}+10$ & 10,83 \\
\hline
\end{tabular}

\section{Isotonic equilibrium neutron density at a temperature of $10^{8} \mathrm{~K}$}

\begin{tabular}{|c|c|c|c|c|c|c|c|c|c|c|c|c|}
\hline & nuc $_{1}$ & $\begin{array}{l}\log y_{1} \\
(S i=6)\end{array}$ & $\sigma_{1}(\mathrm{mb})$ & nuc $_{2}$ & $\mathbf{T}_{2}$ & $\begin{array}{l}\text { time } \\
\text { unit }\end{array}$ & $\sigma_{2}(m b)$ & nuc $_{3}$ & $\sigma_{3}(\mathrm{mb})$ & $\begin{array}{l}\log y_{3} \\
(S i=6)\end{array}$ & $\mathrm{n}_{\mathrm{n}}\left(\mathrm{cm}^{-3}\right)$ & $\lg n_{n}$ \\
\hline 1. & $58 \mathrm{Fe}$ & 3,473 & 13,3 & 44,4495 & d & $59 \mathrm{Fe}$ & 26,4 & $59 \mathrm{Co}$ & 40,1 & 3,352 & $-1,4 \mathrm{E}+10$ & $\mathrm{n} / \mathrm{a}$ \\
\hline 2. & $59 \mathrm{Co}$ & 3,352 & 40,1 & 1925,28 & d & $60 \mathrm{Co}$ & 12,64 & $60 \mathrm{Ni}$ & 29,9 & 4,11 & $-1,2 \mathrm{E}+09$ & $\mathrm{n} / \mathrm{a}$ \\
\hline 3. & $62 \mathrm{Ni}$ & 3,255 & 22,2 & 100,1 & $y$ & $63 \mathrm{Ni}$ & 66,7 & $63 \mathrm{Cu}$ & 53,7 & 2,558 & $1,8 \mathrm{E}+07$ & 7,26 \\
\hline 4. & $64 \mathrm{Ni}$ & 2,726 & 8 & 2,5172 & $h$ & $65 \mathrm{Ni}$ & $\underline{21,2}$ & $65 \mathrm{Cu}$ & 29 & 2,207 & $-1,2 \mathrm{E}+12$ & $\mathrm{n} / \mathrm{a}$ \\
\hline 5. & $65 \mathrm{Cu}$ & 2,207 & 29 & 5,12 & $m$ & $66 \mathrm{Cu}$ & $\underline{126,6}$ & $66 Z n$ & 36,4 & 2,544 & $-4,2 E+13$ & $\mathrm{n} / \mathrm{a}$ \\
\hline 6. & $68 Z n$ & 2,369 & 20,7 & 56,4 & $\mathrm{~m}$ & $69 Z n$ & $\underline{75,4}$ & $69 \mathrm{Ga}$ & 118,7 & 1,358 & $7,9 \mathrm{E}+12$ & 12,90 \\
\hline 7. & $68 Z n$ & 2,369 & 20,7 & 13,76 & $\mathrm{~h}^{*}$ & $69 \mathrm{Zn}^{\mathrm{m}}$ & 110,9 & $69 \mathrm{Ga}$ & 118,7 & 1,358 & $3,7 \mathrm{E}+11$ & 11,56 \\
\hline 8. & $70 \mathrm{Zn}$ & 0,893 & 10,9 & 2,45 & $m$ & $71 Z n$ & $\underline{48,26}$ & $71 \mathrm{Ga}$ & 106,4 & 1,176 & $-3,4 \mathrm{E}+14$ & $\mathrm{n} / \mathrm{a}$ \\
\hline 9. & $70 Z n$ & 0,893 & 10,9 & 3,96 & $h$ & $71 \mathrm{Zn}^{m}$ & $\underline{48,26}$ & $71 \mathrm{Ga}$ & 106,4 & 1,176 & $-3,5 E+12$ & $\mathrm{n} / \mathrm{a}$ \\
\hline 10. & $69 \mathrm{Ga}$ & 1,358 & 118,7 & 21,14 & $\mathrm{~m}$ & $70 \mathrm{Ga}$ & $\underline{301,5}$ & $70 \mathrm{Ge}$ & 89,1 & 1,387 & $1,6 \mathrm{E}+12$ & 12,22 \\
\hline 11. & $71 \mathrm{Ga}$ & 1,176 & 106,4 & 14,095 & $\mathrm{~h}$ & $72 \mathrm{Ga}$ & 267,6 & $72 \mathrm{Ge}$ & 53,1 & 1,513 & $-1,7 \mathrm{E}+10$ & $\mathrm{n} / \mathrm{a}$ \\
\hline 12. & $74 \mathrm{Ge}$ & 1,638 & 37,4 & 82,78 & $\mathrm{~m}$ & $75 \mathrm{Ge}$ & 203,1 & $75 \mathrm{As}$ & 355 & 0,817 & $-7,7 \mathrm{E}+11$ & $\mathrm{n} / \mathrm{a}$ \\
\hline 13. & $75 \mathrm{As}$ & 0,817 & 355 & 1,0942 & d & 76As & 469,6 & $76 \mathrm{Se}$ & 168 & 0,748 & $4,9 \mathrm{E}+11$ & 11,69 \\
\hline 14. & $78 \mathrm{Se}$ & 1,164 & 61,1 & $2,95 E+05$ & $y$ & 79Se & 263 & $79 \mathrm{Br}$ & 622 & 0,775 & $-7,8 \mathrm{E}+03$ & $\mathrm{n} / \mathrm{a}$ \\
\hline 15. & $80 \mathrm{Se}$ & 1,49 & 38 & 18,45 & $\mathrm{~m}$ & $81 \mathrm{Se}$ & $\underline{229}$ & $81 \mathrm{Br}$ & 239 & 0,766 & $-1,6 E+12$ & $\mathrm{n} / \mathrm{a}$ \\
\hline 16. & $80 \mathrm{Se}$ & 1,49 & 38 & 57,28 & $\mathrm{~m}^{*}$ & $81 \mathrm{Se}^{\mathrm{m}}$ & $\underline{229}$ & $81 \mathrm{Br}$ & 239 & 0,766 & $-5,1 \mathrm{E}+11$ & $\mathrm{n} / \mathrm{a}$ \\
\hline 17. & $79 \mathrm{Br}$ & 0,775 & 622 & 17,68 & $\mathrm{~m}$ & $80 \mathrm{Br}$ & $\underline{790}$ & $80 \mathrm{Kr}$ & 274 & 0,009 & $3,6 \mathrm{E}+13$ & 13,56 \\
\hline 18. & $79 \mathrm{Br}$ & 0,775 & 622 & 4,4205 & $\mathrm{~h}^{*}$ & $80 \mathrm{Br}^{\mathrm{m}}$ & $\underline{790}$ & $80 \mathrm{Kr}$ & 90,4 & 0,009 & $7,8 \mathrm{E}+12$ & 12,89 \\
\hline 19. & $81 \mathrm{Br}$ & 0,766 & 239 & 35,282 & $\mathrm{~h}$ & $82 \mathrm{Br}$ & 390,5 & $82 \mathrm{Kr}$ & 93 & 0,716 & $1,0 \mathrm{E}+11$ & 11,00 \\
\hline 20. & $84 \mathrm{Kr}$ & 1,408 & 32,6 & 3916,8 & $d$ & $85 \mathrm{Kr}$ & 73 & $85 \mathrm{Rb}$ & 234 & 0,709 & $-3,1 \mathrm{E}+07$ & $\mathrm{n} / \mathrm{a}$ \\
\hline 21. & $84 \mathrm{Kr}$ & 1,408 & 32,6 & 4,78 & h79\% & $85 \mathrm{Kr}^{\mathrm{m}}$ & 73 & $85 \mathrm{Rb}$ & 234 & 0,709 & $-6,2 \mathrm{E}+11$ & $\mathrm{n} / \mathrm{a}$ \\
\hline 22. & $86 \mathrm{Kr}$ & 0,893 & 4,76 & 76,3 & $\mathrm{~m}$ & $87 \mathrm{Kr}$ & $\underline{18}$ & $87 \mathrm{Rb}$ & 15,7 & 0,316 & $4,5 \mathrm{E}+12$ & 12,65 \\
\hline 23. & $85 \mathrm{Rb}$ & 0,709 & 234 & 18,642 & d & $86 \mathrm{Rb}$ & 202 & $86 \mathrm{Sr}$ & 63,5 & 0,365 & $5,6 \mathrm{E}+10$ & 10,75 \\
\hline 24. & $87 \mathrm{Rb}$ & 0,316 & 15,7 & 17,773 & $\mathrm{~m}$ & $88 \mathrm{Rb}$ & $\underline{110}$ & $88 \mathrm{Sr}$ & 6,16 & 1,288 & $-1,5 E+13$ & $\mathrm{n} / \mathrm{a}$ \\
\hline 25. & $88 \mathrm{Sr}$ & 1,288 & 6,16 & 50,57 & d & $89 S r$ & 19 & $89 Y$ & 19,3 & 0,667 & $1,0 \mathrm{E}+10$ & 10,01 \\
\hline
\end{tabular}




\begin{tabular}{|c|c|c|c|c|c|c|c|c|c|c|c|c|}
\hline & nuc $_{1}$ & $\begin{array}{l}\log y_{1} \\
(S i=6)\end{array}$ & $\sigma_{1}(\mathrm{mb})$ & nuc $_{2}$ & $T_{2}$ & $\begin{array}{l}\text { time } \\
\text { unit }\end{array}$ & $\sigma_{2}(\mathrm{mb})$ & nuc $_{3}$ & $\sigma_{3}(\mathrm{mb})$ & $\begin{array}{l}\log y_{3} \\
(S i=6)\end{array}$ & $\mathrm{n}_{\mathrm{n}}\left(\mathrm{cm}^{-3}\right)$ & $\lg n_{n}$ \\
\hline 26. & $89 \mathrm{Y}$ & 0,667 & 19,3 & 90 & $y$ & $90 \mathrm{Y}$ & 149,9 & $90 Z r$ & 19,3 & 0,769 & $-1,3 E+06$ & $\mathrm{n} / \mathrm{a}$ \\
\hline 27. & $92 Z r$ & 0,299 & 37,8 & $1,53 E+06$ & $y$ & $93 Z r$ & 96 & $93 \mathrm{Nb}$ & 265,7 & 0,299 & $-4,4 \mathrm{E}+02$ & $n / a$ \\
\hline 28. & $93 \mathrm{Nb}$ & $-0,156$ & 265,7 & $2,03 E+04$ & $y$ & $94 \mathrm{Nb}$ & 482 & $94 \mathrm{Mo}$ & 109,6 & $-0,636$ & $2,0 \mathrm{E}+09$ & 9,30 \\
\hline 29. & $102 R u$ & $-0,231$ & 151 & 39,26 & $d$ & $103 R u$ & 343 & $103 \mathrm{Rh}$ & 810 & $-0,463$ & $-3,2 \mathrm{E}+10$ & $\mathrm{n} / \mathrm{a}$ \\
\hline 30. & $103 \mathrm{Rh}$ & $-0,463$ & 810 & 42,3 & $\mathrm{~s}$ & 104Rh & 154 & $104 \mathrm{Pd}$ & 274 & $-0,818$ & $2,2 \mathrm{E}+15$ & 15,35 \\
\hline 31. & $103 \mathrm{Rh}$ & $-0,463$ & 810 & 4,34 & $\mathrm{~m}^{*}$ & $104 \mathrm{Rh}^{\mathrm{m}}$ & 154 & $104 \mathrm{Pd}$ & 274 & $-0,818$ & $3,6 \mathrm{E}+14$ & 14,56 \\
\hline 32. & $106 \mathrm{Pd}$ & $-0,42$ & 244 & $6,60 \mathrm{E}+06$ & $y$ & $107 \mathrm{Pd}$ & 1302 & $107 \mathrm{Ag}$ & 787 & $-0,602$ & $-5,7 \mathrm{E}+00$ & $\mathrm{n} / \mathrm{a}$ \\
\hline 33. & $108 \mathrm{Pd}$ & $-0,431$ & 218 & 13,7012 & $\mathrm{~h}$ & $109 \mathrm{Pd}$ & 236,3 & $109 \mathrm{Ag}$ & 793 & $-0,627$ & $-1,2 \mathrm{E}+11$ & $\mathrm{n} / \mathrm{a}$ \\
\hline 34. & $107 \mathrm{Ag}$ & $-0,602$ & 787 & 2,37 & $\mathrm{~m}$ & $108 \mathrm{Ag}$ & 1788 & $108 \mathrm{Cd}$ & 202 & $-1,851$ & $6,8 \mathrm{E}+14$ & 14,84 \\
\hline 35. & $107 \mathrm{Ag}$ & $-0,602$ & 787 & 438 & $y^{*}$ & $108 \mathrm{Ag}^{\mathrm{m}}$ & 1383,0 & $108 \mathrm{Cd}$ & 202 & $-1,851$ & $9,1 \mathrm{E}+06$ & 6,96 \\
\hline 36. & $109 \mathrm{Ag}$ & $-0,627$ & 793 & 24,6 & $\mathrm{~s}$ & $110 \mathrm{Ag}$ & 1172 & $110 \mathrm{Cd}$ & 229,9 & $-0,701$ & 1,1E+14 & 14,05 \\
\hline 37. & $109 \mathrm{Ag}$ & $-0,627$ & 793 & 249,76 & $d$ & $110 \mathrm{Ag}^{\mathrm{m}}$ & 1172 & $110 \mathrm{Cd}$ & 229,9 & $-0,701$ & $1,3 \mathrm{E}+08$ & 8,11 \\
\hline 38. & $114 \mathrm{Cd}$ & $-0,333$ & 135,3 & 53,46 & $\mathrm{~h}$ & $115 \mathrm{Cd}$ & 290 & $115 \ln$ & 776 & $-0,754$ & $-2,5 E+10$ & $\mathrm{n} / \mathrm{a}$ \\
\hline 39. & $114 \mathrm{Cd}$ & $-0,333$ & 135,3 & 44,56 & $d^{*}$ & $115 \mathrm{Cd}^{\mathrm{m}}$ & 224 & 115In & 776 & $-0,754$ & $-1,6 \mathrm{E}+09$ & $\mathrm{n} / \mathrm{a}$ \\
\hline 40. & $113 \ln$ & $-2,103$ & 229 & 71,9 & $\mathrm{~s}$ & $114 \ln$ & $\underline{1308}$ & $114 \mathrm{Sn}$ & 134,4 & $-1,599$ & $-1,3 E+13$ & $\mathrm{n} / \mathrm{a}$ \\
\hline 41. & $113 \mathrm{In}$ & $-2,103$ & 580 & 49,51 & $d^{*}$ & $114 \mathrm{In}^{\mathrm{m}}$ & 2595 & $114 \mathrm{Sn}$ & 134,4 & $-1,599$ & $8,1 \mathrm{E}+07$ & 7,91 \\
\hline 42. & $115 \ln$ & $-0,754$ & 154 & 14,1 & $\mathrm{~s}$ & $116 \mathrm{In}$ & 1377 & $116 \mathrm{Sn}$ & 92,3 & $-0,263$ & $-6,1 \mathrm{E}+13$ & $\mathrm{n} / \mathrm{a}$ \\
\hline 43. & $115 \ln$ & $-0,754$ & 622 & 54,29 & $\mathrm{~m}$ & $116 \mathrm{In}^{\mathrm{m}}$ & 1377 & $116 \mathrm{Sn}$ & 92,3 & $-0,263$ & $6,7 \mathrm{E}+11$ & 11,83 \\
\hline 44. & $120 S n$ & 0,097 & 36,3 & 27,03 & $\mathrm{~h}$ & $121 S n$ & 167 & $121 \mathrm{Sb}$ & 532 & $-0,752$ & $-3,3 E+10$ & $\mathrm{n} / \mathrm{a}$ \\
\hline 45. & $120 \mathrm{Sn}$ & 0,097 & 36,3 & 43,9 & $y^{*}$ & $121 \mathrm{Sn}^{\mathrm{m}}$ & 175,4 & $121 \mathrm{Sb}$ & 532 & $-0,752$ & $-2,2 \mathrm{E}+06$ & $\mathrm{n} / \mathrm{a}$ \\
\hline 46. & $122 \mathrm{Sn}$ & $-0,745$ & 22,6 & 129,2 & d & $123 S n$ & 361 & $123 \mathrm{Sb}$ & 303 & $-0,879$ & $-4,0 \mathrm{E}+10$ & $\mathrm{n} / \mathrm{a}$ \\
\hline 47. & $122 \mathrm{Sn}$ & $-0,745$ & 21,7 & 40,06 & $\mathrm{~m}$ & $123 \mathrm{Sn}^{\mathrm{m}}$ & 361 & $123 \mathrm{Sb}$ & 303 & $-0,879$ & $-1,9 \mathrm{E}+14$ & $\mathrm{n} / \mathrm{a}$ \\
\hline 48. & $121 \mathrm{Sb}$ & $-0,752$ & 532 & 2,7238 & d & $122 \mathrm{Sb}$ & 894 & $122 \mathrm{Te}$ & 295,4 & $-0,928$ & $2,1 \mathrm{E}+10$ & 10,31 \\
\hline 49. & $123 \mathrm{Sb}$ & $-0,879$ & 303 & 60,11 & d & $124 S b$ & 924,0 & $124 \mathrm{Te}$ & 155 & $-0,654$ & $3,1 \mathrm{E}+11$ & 11,49 \\
\hline 50. & $126 \mathrm{Te}$ & $-0,046$ & 81,3 & 9,35 & $\mathrm{~h}$ & $127 \mathrm{Te}$ & 256,8 & $127 \mid$ & 662 & $-0,046$ & $-2,6 \mathrm{E}+11$ & $\mathrm{n} / \mathrm{a}$ \\
\hline 51. & $126 \mathrm{Te}$ & $-0,046$ & 81,3 & 109 & $d^{*}$ & $127 \mathrm{Te}^{\mathrm{m}}$ & 668,6 & $127 \mid$ & 662 & $-0,046$ & $-3,6 \mathrm{E}+08$ & $\mathrm{n} / \mathrm{a}$ \\
\hline 52. & $127 \mid$ & $-0,046$ & 662 & 24,99 & $\mathrm{~m}$ & $128 I$ & 679,5 & $128 X e$ & 262,5 & 0,991 & $-1,8 \mathrm{E}+12$ & $\mathrm{n} / \mathrm{a}$ \\
\hline 53. & $132 X e$ & 0,086 & 63,8 & 5,243 & $d$ & $133 \times e$ & 127 & $133 \mathrm{Cs}$ & 502 & $-0,429$ & $-2,7 E+10$ & $\mathrm{n} / \mathrm{a}$ \\
\hline 54. & $133 \mathrm{Cs}$ & $-0,429$ & 502 & 2,0652 & $y$ & $134 \mathrm{Cs}$ & 724 & 134Ba & 176 & $-0,963$ & $1,5 \mathrm{E}+09$ & 9,17 \\
\hline 55. & $138 \mathrm{Ba}$ & 0,508 & 4,13 & 83,06 & $\mathrm{~m}$ & 139Ba & 39,7 & 139La & 32,4 & $-0,351$ & $-1,0 \mathrm{E}+12$ & $\mathrm{n} / \mathrm{a}$ \\
\hline 56. & 139La & $-0,351$ & 32,4 & 1,67855 & $d$ & 140La & 117,8 & $140 \mathrm{Ce}$ & 11,73 & 0,004 & $3,3 E+10$ & 10,52 \\
\hline 57. & $140 \mathrm{Ce}$ & 0,004 & 11,73 & 32,508 & $d$ & $141 \mathrm{Ce}$ & 76 & $141 \mathrm{Pr}$ & 111,4 & $-0,777$ & $-4,4 \mathrm{E}+09$ & $\mathrm{n} / \mathrm{a}$ \\
\hline 58. & $141 \mathrm{Pr}$ & $-0,777$ & 111,4 & 19,12 & $\mathrm{~h}$ & 1 & 361,4 & $142 \mathrm{Nd}$ & 1 & $-0,65$ & $5,4 \mathrm{E}+10$ & 10,73 \\
\hline 59. & $150 \mathrm{Sm}$ & $-1,717$ & 422,3 & 90 & $y$ & $151 \mathrm{Sm}$ & 3040 & $151 \mathrm{Eu}$ & 3556 & 0,35 & $-2,8 E+05$ & $\mathrm{n} / \mathrm{a}$ \\
\hline 60. & $152 \mathrm{Sm}$ & $-1,161$ & 464,8 & 46,284 & $\mathrm{~h}$ & $153 \mathrm{Sm}$ & 1095 & 153Eu & 2567 & $-1,294$ & $-1,2 \mathrm{E}+10$ & $\mathrm{n} / \mathrm{a}$ \\
\hline 61. & 153Eu & $-1,294$ & 2557 & 8,59 & $\mathrm{y}$ & $154 \mathrm{Eu}$ & 4420 & $154 \mathrm{Gd}$ & 1028 & $-2,149$ & $8,8 \mathrm{E}+07$ & 7,95 \\
\hline 62. & $158 \mathrm{Gd}$ & $-1,086$ & 323,6 & 18,479 & $\mathrm{~h}$ & $159 \mathrm{Gd}$ & 455,2 & $159 \mathrm{~Tb}$ & 1817 & $-1,22$ & $-6,3 E+10$ & $\mathrm{n} / \mathrm{a}$ \\
\hline
\end{tabular}




\begin{tabular}{|c|c|c|c|c|c|c|c|c|c|c|c|c|}
\hline & nuc $_{1}$ & $\begin{array}{l}\log y_{1} \\
(S i=6)\end{array}$ & $\sigma_{1}(\mathrm{mb})$ & nuc $_{2}$ & $T_{2}$ & $\begin{array}{l}\text { time } \\
\text { unit }\end{array}$ & $\sigma_{2}(\mathrm{mb})$ & $\mathrm{nuc}_{3}$ & $\sigma_{3}(\mathrm{mb})$ & $\begin{array}{l}\log y_{3} \\
(S i=6)\end{array}$ & $\mathrm{n}_{\mathrm{n}}\left(\mathrm{cm}^{-3}\right)$ & $\lg n_{n}$ \\
\hline 63. & $159 \mathrm{~Tb}$ & $-1,22$ & 1817 & 72,3 & $d$ & $160 \mathrm{~Tb}$ & 3240 & 160Dy & 890 & $-2,044$ & $3,6 \mathrm{E}+09$ & 9,55 \\
\hline 64. & 164Dy & $-0,955$ & 212 & 2,334 & $\mathrm{~h}$ & 165Dy & 284,5 & $165 \mathrm{Ho}$ & 1237 & $-1,051$ & $-8,4 \mathrm{E}+11$ & $\mathrm{n} / \mathrm{a}$ \\
\hline 65. & $165 \mathrm{Ho}$ & $-1,051$ & 1237 & 26,83 & $\mathrm{~h}$ & $166 \mathrm{Ho}$ & 1262,0 & $166 \mathrm{Er}$ & 700 & $-1,077$ & $2,1 \mathrm{E}+09$ & 9,32 \\
\hline 66. & $165 \mathrm{Ho}$ & $-1,051$ & 1237 & $1,20 E+03$ & $y$ & $166 \mathrm{Ho}^{\mathrm{m}}$ & 1235,3 & $166 \mathrm{Er}$ & 700 & $-1,077$ & $5,4 \mathrm{E}+03$ & 3,73 \\
\hline 67. & $168 \mathrm{Er}$ & $-1,168$ & 319 & 9,392 & $d$ & $169 \mathrm{Er}$ & 653 & 169Tm & 1065 & $-1,423$ & $-2,5 E+09$ & $\mathrm{n} / \mathrm{a}$ \\
\hline 68. & $169 \mathrm{Tm}$ & $-1,423$ & 1065 & 128,6 & $\mathrm{~d}$ & $170 \mathrm{Tm}$ & 1870 & $170 \mathrm{Yb}$ & 768,3 & $-2,124$ & $7,2 \mathrm{E}+08$ & 8,86 \\
\hline 69. & $174 \mathrm{Yb}$ & $-1,103$ & 150,5 & 4,158 & $d$ & $175 \mathrm{Yb}$ & 558 & $175 \mathrm{Lu}$ & 1219 & $-1,449$ & $-1,1 E+10$ & $\mathrm{n} / \mathrm{a}$ \\
\hline 70. & 176Lu & $-2,959$ & 1639 & 6,6475 & $\mathrm{~d}$ & 177Lu & 794,9 & $177 \mathrm{Hf}$ & 1544 & $-1,545$ & $-5,8 \mathrm{E}+09$ & $\mathrm{n} / \mathrm{a}$ \\
\hline 71. & $176 \mathrm{Lu}$ & $-2,959$ & 1639 & 160,44 & $d$ & $177 \mathrm{Lu}^{\mathrm{m}}$ & 348,3 & $177 \mathrm{Hf}$ & 1544 & $-1,545$ & $-5,5 E+08$ & $\mathrm{n} / \mathrm{a}$ \\
\hline 72. & $176 \mathrm{Lu}$ & $-2,959$ & 1639 & 6 & $\mathrm{~m}$ & $177 \mathrm{Lu}^{\mathrm{m}}$ & 348,3 & $177 \mathrm{Hf}$ & 1544 & $-1,545$ & $-2,1 \mathrm{E}+13$ & $\mathrm{n} / \mathrm{a}$ \\
\hline 73. & $180 \mathrm{Hf}$ & $-1,265$ & 156,6 & 42,39 & $d$ & $181 \mathrm{Hf}$ & 194 & $181 \mathrm{Ta}$ & 766 & $-1,684$ & $-2,2 E+09$ & $\mathrm{n} / \mathrm{a}$ \\
\hline 74. & $181 \mathrm{Ta}$ & $-1,684$ & 766 & 114,43 & $\mathrm{~d}$ & $182 \mathrm{Ta}$ & 1120 & $182 \mathrm{~W}$ & 285 & $-1,455$ & $3,0 \mathrm{E}+06$ & 6,48 \\
\hline 75. & $184 W$ & $-1,389$ & 225 & 75,4 & $d$ & $185 \mathrm{~W}$ & 633 & $185 \mathrm{Re}$ & 1438,5 & $-1,717$ & $-4,5 E+08$ & $\mathrm{n} / \mathrm{a}$ \\
\hline 76. & $186 W$ & $-1,423$ & 226 & 23,72 & $\mathrm{~h}$ & $187 \mathrm{~W}$ & $\underline{183}$ & $187 \mathrm{Re}$ & 1184 & $-1,475$ & $-1,3 E+11$ & $\mathrm{n} / \mathrm{a}$ \\
\hline 77. & $185 \mathrm{Re}$ & $-1,717$ & 1420 & 3,7186 & $d$ & $186 \operatorname{Re}$ & 743 & $1860 s$ & 414 & $-2,06$ & $7,0 \mathrm{E}+10$ & 10,85 \\
\hline 78. & $185 \mathrm{Re}$ & $-1,717$ & 18,5 & $2,00 E+05$ & $\mathrm{y}^{*}$ & $186 \operatorname{Re}^{m}$ & 743 & $1860 \mathrm{~s}$ & 414 & $-2,06$ & $-4,9 \mathrm{E}+02$ & $n / a$ \\
\hline 79. & $187 \operatorname{Re}$ & $-1,475$ & 1187 & 17,003 & $\mathrm{~h}$ & $188 \mathrm{Re}$ & $\underline{2343}$ & $1880 \mathrm{~s}$ & 294 & $-1,047$ & $9,0 \mathrm{E}+09$ & 9,96 \\
\hline 80. & $1900 \mathrm{~s}$ & $-0,75$ & 278 & 15,4 & $d$ & 1910s & 1290 & $191 / r$ & 1350 & $-0,607$ & $-1,4 \mathrm{E}+09$ & $\mathrm{n} / \mathrm{a}$ \\
\hline 81. & $1920 s$ & $-0,558$ & 160 & 30,11 & $\mathrm{~h}$ & $1930 s$ & 95,6 & $193 \mathrm{lr}$ & 994 & $-0,383$ & $-2,2 E+11$ & $\mathrm{n} / \mathrm{a}$ \\
\hline 82. & $191 \mathrm{Ir}$ & $-0,607$ & 1350 & $7,38 \mathrm{E}+01$ & $d$ & $192 \mathrm{Ir}$ & 2080 & $192 \mathrm{Pt}$ & 483 & $-1,979$ & $1,3 E+10$ & 10,10 \\
\hline 83. & $191 / r$ & $-0,607$ & 1350 & $2,41 \mathrm{E}+02$ & $\mathrm{y}^{*}$ & $192 \mathrm{Ir}^{\mathrm{m}}$ & 2080 & $192 \mathrm{Pt}$ & 483 & $-1,979$ & $1,1 \mathrm{E}+07$ & 7,02 \\
\hline 84. & $193 \mathrm{Ir}$ & $-0,383$ & 994 & 19,28 & $\mathrm{~h}$ & $194 \mathrm{Ir}$ & 397,8 & $194 \mathrm{Pt}$ & 283 & $-0,356$ & $2,2 \mathrm{E}+11$ & 11,34 \\
\hline 85. & $196 \mathrm{Pt}$ & $-0,47$ & 167,4 & 19,8915 & $\mathrm{~h}$ & $197 \mathrm{Pt}$ & 175,4 & $197 \mathrm{Au}$ & 612,8 & $-0,728$ & $-1,0 \mathrm{E}+11$ & $\mathrm{n} / \mathrm{a}$ \\
\hline 86. & $197 \mathrm{Au}$ & $-0,728$ & 612,8 & $2,70 \mathrm{E}+00$ & $\mathrm{~d}$ & 198Au & 840 & $198 \mathrm{Hg}$ & 173 & $-1,46$ & $2,5 \mathrm{E}+11$ & 11,39 \\
\hline 87. & $202 \mathrm{Hg}$ & $-0,996$ & 63,3 & 46,594 & $d$ & $203 \mathrm{Hg}$ & 98 & $203 \mathrm{TI}$ & 170,5 & $-1,265$ & $-9,8 E+10$ & $\mathrm{n} / \mathrm{a}$ \\
\hline 88. & $204 \mathrm{Hg}$ & $-1,633$ & 42 & 5,14 & $\mathrm{~m}$ & $205 \mathrm{Hg}$ & $\underline{12,7}$ & 205TI & 52,6 & $-0,886$ & $-5,6 E+14$ & $\mathrm{n} / \mathrm{a}$ \\
\hline 89. & 203TI & $-1,265$ & 170,5 & 3,78 & $y$ & $204 \mathrm{TI}$ & 215 & $204 \mathrm{~Pb}$ & 83,7 & $-1,207$ & $7,8 \mathrm{E}+07$ & 7,89 \\
\hline 90. & $205 \mathrm{TI}$ & $-0,866$ & 52,6 & 4,2 & $\mathrm{~m}$ & $206 \mathrm{TI}$ & $\underline{34,1}$ & $206 \mathrm{~Pb}$ & 14,7 & $-0,277$ & $-2,3 E+13$ & $\mathrm{n} / \mathrm{a}$ \\
\hline 91. & $208 \mathrm{~Pb}$ & 0,265 & 0,376 & 3,253 & $\mathrm{~h}$ & $209 \mathrm{~Pb}$ & 3,6 & $209 \mathrm{Bi}$ & 2,61 & $-0,842$ & $5,1 \mathrm{E}+13$ & 13,71 \\
\hline
\end{tabular}

\section{Isotonic equilibrium neutron density at a temperature of $3 \cdot 10^{8} \mathrm{~K}$}

\begin{tabular}{|c|c|c|c|c|c|c|c|c|c|c|c|c|}
\hline & nuc $_{1}$ & $\begin{array}{l}\log y_{1} \\
(S i=6)\end{array}$ & $\sigma_{1}(\mathrm{mb}$ & $\mathrm{T}_{2}$ & $\begin{array}{l}\text { time } \\
\text { unit }\end{array}$ & nuc $_{2}$ & $\sigma_{2}(m b)$ & nuc $_{3}$ & $\sigma_{3}(\mathrm{mb})$ & $\begin{array}{l}\log y_{3} \\
(S i=6)\end{array}$ & $\mathrm{n}_{\mathrm{n}}\left(\mathrm{cm}^{-3}\right)$ & $\lg n_{n}$ \\
\hline 1. & $58 \mathrm{Fe}$ & 3,473 & 13,3 & 44,4495 & $d$ & $59 \mathrm{Fe}$ & 26,4 & 59 Co & 40,1 & 3,352 & $-1,41 E+10$ & $\mathrm{n} / \mathrm{a}$ \\
\hline 2. & $59 \mathrm{Co}$ & 3,352 & 40,1 & 1925,28 & d & $60 \mathrm{Co}$ & 12,64 & $60 \mathrm{Ni}$ & 29,9 & 4,11 & $-2,85 E+10$ & $\mathrm{n} / \mathrm{a}$ \\
\hline 3. & $62 \mathrm{Ni}$ & 3,255 & 22,2 & 100,1 & $y$ & $63 \mathrm{Ni}$ & 66,7 & $63 \mathrm{Cu}$ & 53,7 & 2,558 & $9,50 \mathrm{E}+07$ & 7,98 \\
\hline
\end{tabular}




\begin{tabular}{|c|c|c|c|c|c|c|c|c|c|c|c|c|}
\hline & nuc $_{1}$ & $\begin{array}{l}\log y_{1} \\
(S i=6)\end{array}$ & $\sigma_{1}(\mathrm{mb}$ & $T_{2}$ & $\begin{array}{l}\text { time } \\
\text { unit }\end{array}$ & nuc $_{2}$ & $\sigma_{2}(\mathrm{mb})$ & nuc $_{3}$ & $\sigma_{3}(\mathrm{mb})$ & $\begin{array}{l}\log y_{3} \\
(S i=6)\end{array}$ & $\mathrm{n}_{\mathrm{n}}\left(\mathrm{cm}^{-3}\right)$ & $\lg n_{n}$ \\
\hline 4. & $64 \mathrm{Ni}$ & 2,726 & 8 & 2,5172 & $h$ & $65 \mathrm{Ni}$ & $\underline{21,2}$ & $65 \mathrm{Cu}$ & 29 & 2,207 & $-1,18 \mathrm{E}+12$ & $\mathrm{n} / \mathrm{a}$ \\
\hline 5. & $65 \mathrm{Cu}$ & 2,207 & 29 & 5,12 & $m$ & $66 \mathrm{Cu}$ & $\underline{126,6}$ & $66 Z n$ & 36,4 & 2,544 & $-4,14 \mathrm{E}+13$ & $\mathrm{n} / \mathrm{a}$ \\
\hline 6. & $68 Z n$ & 2,369 & 20,7 & 56,4 & $\mathrm{~m}$ & $69 Z n$ & $\underline{75,4}$ & $69 \mathrm{Ga}$ & 118,7 & 1,358 & $7,89 \mathrm{E}+12$ & 12,90 \\
\hline 7. & $68 Z n$ & 2,369 & 20,7 & 13,76 & $\mathrm{~h}^{*}$ & $69 \mathrm{Zn}^{\mathrm{m}}$ & 110,9 & $69 \mathrm{Ga}$ & 118,7 & 1,358 & $3,67 E+11$ & 11,56 \\
\hline 8. & $70 \mathrm{Zn}$ & 0,893 & 10,9 & 2,45 & $m$ & $71 \mathrm{Zn}$ & $\underline{48,26}$ & $71 \mathrm{Ga}$ & 106,4 & 1,176 & $-3,37 E+14$ & $\mathrm{n} / \mathrm{a}$ \\
\hline 9. & $70 \mathrm{Zn}$ & 0,893 & 10,9 & 3,96 & $h$ & $71 \mathrm{Zn}^{m}$ & $\underline{48,26}$ & $71 \mathrm{Ga}$ & 106,4 & 1,176 & $-3,48 E+12$ & $\mathrm{n} / \mathrm{a}$ \\
\hline 10. & $69 \mathrm{Ga}$ & 1,358 & 118,7 & 21,14 & $\mathrm{~m}$ & $70 \mathrm{Ga}$ & $\underline{301,5}$ & $70 \mathrm{Ge}$ & 89,1 & 1,387 & $1,64 \mathrm{E}+12$ & 12,22 \\
\hline 11. & $71 \mathrm{Ga}$ & 1,176 & 106,4 & 14,095 & $\mathrm{~h}$ & $72 \mathrm{Ga}$ & 267,6 & $72 \mathrm{Ge}$ & 53,1 & 1,513 & $-7,81 E+10$ & $\mathrm{n} / \mathrm{a}$ \\
\hline 12. & $74 \mathrm{Ge}$ & 1,638 & 37,4 & 82,78 & $\mathrm{~m}$ & $75 \mathrm{Ge}$ & 203,1 & 75As & 355 & 0,817 & $-7,49 E+11$ & $\mathrm{n} / \mathrm{a}$ \\
\hline 13. & 75 As & 0,817 & 355 & 1,0942 & $d$ & 76As & 469,6 & $76 \mathrm{Se}$ & 168 & 0,748 & $1,40 \mathrm{E}+13$ & 13,15 \\
\hline 14. & $78 \mathrm{Se}$ & 1,164 & 61,1 & $2,95 \mathrm{E}+05$ & $y$ & $79 S e$ & 263 & $79 B r$ & 622 & 0,775 & $-1,39 E+07$ & $\mathrm{n} / \mathrm{a}$ \\
\hline 15. & $80 \mathrm{Se}$ & 1,49 & 38 & 18,45 & $\mathrm{~m}$ & $81 \mathrm{Se}$ & $\underline{229}$ & $81 \mathrm{Br}$ & 239 & 0,766 & $-1,48 E+12$ & $\mathrm{n} / \mathrm{a}$ \\
\hline 16. & $80 \mathrm{Se}$ & 1,49 & 38 & 57,28 & $\mathrm{~m}^{*}$ & $81 \mathrm{Se}^{\mathrm{m}}$ & $\underline{229}$ & $81 \mathrm{Br}$ & 239 & 0,766 & $-4,78 \mathrm{E}+11$ & $\mathrm{n} / \mathrm{a}$ \\
\hline 17. & $79 \mathrm{Br}$ & 0,775 & 622 & 17,68 & $\mathrm{~m}$ & $80 \mathrm{Br}$ & $\underline{790}$ & $80 \mathrm{Kr}$ & 274 & 0,009 & $2,42 E+13$ & 13,38 \\
\hline 18. & $79 \mathrm{Br}$ & 0,775 & 622 & 4,4205 & $h^{*}$ & $80 \mathrm{Br}^{\mathrm{m}}$ & $\underline{790}$ & $80 \mathrm{Kr}$ & 90,4 & 0,009 & $5,15 \mathrm{E}+12$ & 12,71 \\
\hline 19. & $81 \mathrm{Br}$ & 0,766 & 239 & 35,282 & $\mathrm{~h}$ & $82 \mathrm{Br}$ & 390,5 & $82 \mathrm{Kr}$ & 93 & 0,716 & $1,05 \mathrm{E}+12$ & 12,02 \\
\hline 20. & $84 \mathrm{Kr}$ & 1,408 & 32,6 & 3916,8 & $d$ & $85 \mathrm{Kr}$ & 73 & $85 \mathrm{Rb}$ & 234 & 0,709 & $-3,23 E+07$ & $\mathrm{n} / \mathrm{a}$ \\
\hline 21. & $84 \mathrm{Kr}$ & 1,408 & 32,6 & 4,78 & h79\% & $85 \mathrm{Kr}^{\mathrm{m}}$ & 73 & $85 \mathrm{Rb}$ & 234 & 0,709 & $-6,35 E+11$ & $\mathrm{n} / \mathrm{a}$ \\
\hline 22. & $86 \mathrm{Kr}$ & 0,893 & 4,76 & 76,3 & $\mathrm{~m}$ & $87 \mathrm{Kr}$ & $\underline{18}$ & $87 \mathrm{Rb}$ & 15,7 & 0,316 & $4,48 \mathrm{E}+12$ & 12,65 \\
\hline 23. & $85 \mathrm{Rb}$ & 0,709 & 234 & 18,642 & $d$ & $86 \mathrm{Rb}$ & 202 & $86 \mathrm{Sr}$ & 63,5 & 0,365 & $5,60 \mathrm{E}+10$ & 10,75 \\
\hline 24. & $87 \mathrm{Rb}$ & 0,316 & 15,7 & 17,773 & $\mathrm{~m}$ & $88 \mathrm{Rb}$ & $\underline{110}$ & $88 \mathrm{Sr}$ & 6,16 & 1,288 & $-1,14 \mathrm{E}+13$ & $\mathrm{n} / \mathrm{a}$ \\
\hline 25. & $88 \mathrm{Sr}$ & 1,288 & 6,16 & 50,57 & $d$ & $89 S r$ & 19 & $89 Y$ & 19,3 & 0,667 & $1,03 \mathrm{E}+10$ & 10,01 \\
\hline 26. & $89 Y$ & 0,667 & 19,3 & 90 & $y$ & $90 \mathrm{Y}$ & 149,9 & $90 Z r$ & 19,3 & 0,769 & $-1,26 \mathrm{E}+06$ & $\mathrm{n} / \mathrm{a}$ \\
\hline 27. & $92 Z r$ & 0,299 & 37,8 & $1,53 \mathrm{E}+06$ & $y$ & $93 \mathrm{Zr}$ & 96 & $93 \mathrm{Nb}$ & 265,7 & 0,299 & $-1,58 \mathrm{E}+03$ & $\mathrm{n} / \mathrm{a}$ \\
\hline 28. & $93 \mathrm{Nb}$ & $-0,156$ & 265,7 & $2,03 E+04$ & $y$ & $94 \mathrm{Nb}$ & 482 & $94 \mathrm{Mo}$ & 109,6 & $-0,636$ & $4,04 \mathrm{E}+10$ & 10,61 \\
\hline 29. & $102 \mathrm{Ru}$ & $-0,231$ & 151 & 39,26 & $d$ & 103Ru & 343 & $103 \mathrm{Rh}$ & 810 & $-0,463$ & $-3,59 E+10$ & $\mathrm{n} / \mathrm{a}$ \\
\hline 30. & $103 \mathrm{Rh}$ & $-0,463$ & 810 & 42,3 & $\mathrm{~s}$ & 104Rh & 154 & $104 \mathrm{Pd}$ & 274 & $-0,818$ & $1,73 \mathrm{E}+15$ & 15,24 \\
\hline 31. & 103Rh & $-0,463$ & 810 & 4,34 & $\mathrm{~m}^{*}$ & $104 \mathrm{Rh}^{\mathrm{m}}$ & 154 & $104 \mathrm{Pd}$ & 274 & $-0,818$ & $2,80 \mathrm{E}+14$ & 14,45 \\
\hline 32. & $106 \mathrm{Pd}$ & $-0,42$ & 244 & $6,60 \mathrm{E}+06$ & $y$ & $107 \mathrm{Pd}$ & 1302 & $107 \mathrm{Ag}$ & 787 & $-0,602$ & $-4,02 E+04$ & $\mathrm{n} / \mathrm{a}$ \\
\hline 33. & $108 \mathrm{Pd}$ & $-0,431$ & 218 & 13,7012 & $\mathrm{~h}$ & $109 \mathrm{Pd}$ & 236,3 & $109 \mathrm{Ag}$ & 793 & $-0,627$ & $-1,24 \mathrm{E}+11$ & $\mathrm{n} / \mathrm{a}$ \\
\hline 34. & $107 \mathrm{Ag}$ & $-0,602$ & 787 & 2,37 & $\mathrm{~m}$ & $108 \mathrm{Ag}$ & $\underline{1788}$ & $108 \mathrm{Cd}$ & 202 & $-1,851$ & $5,96 \mathrm{E}+14$ & 14,77 \\
\hline 35. & $107 \mathrm{Ag}$ & $-0,602$ & 787 & 438 & $y^{*}$ & $108 \mathrm{Ag}^{\mathrm{m}}$ & 1383,0 & $108 \mathrm{Cd}$ & 202 & $-1,851$ & $7,92 \mathrm{E}+06$ & 6,90 \\
\hline 36. & $109 \mathrm{Ag}$ & $-0,627$ & 793 & 24,6 & $\mathrm{~s}$ & $110 \mathrm{Ag}$ & 1172 & $110 \mathrm{Cd}$ & 229,9 & $-0,701$ & $1,03 E+14$ & 14,01 \\
\hline 37. & $109 \mathrm{Ag}$ & $-0,627$ & 793 & 249,76 & $d$ & $110 \mathrm{Ag}^{\mathrm{m}}$ & 1172 & $110 \mathrm{Cd}$ & 229,9 & $-0,701$ & $1,18 \mathrm{E}+08$ & 8,07 \\
\hline 38. & $114 \mathrm{Cd}$ & $-0,333$ & 135,3 & 53,46 & $\mathrm{~h}$ & $115 \mathrm{Cd}$ & 290 & $115 \ln$ & 776 & $-0,754$ & $-2,47 E+10$ & $\mathrm{n} / \mathrm{a}$ \\
\hline
\end{tabular}




\begin{tabular}{|c|c|c|c|c|c|c|c|c|c|c|c|c|}
\hline & nuc $_{1}$ & $\begin{array}{l}\log y_{1} \\
(S i=6)\end{array}$ & $\sigma_{1}(\mathrm{mb}$ & $T_{2}$ & $\begin{array}{l}\text { time } \\
\text { unit }\end{array}$ & nuc $_{2}$ & $\sigma_{2}(\mathbf{m b})$ & nuc $_{3}$ & $\sigma_{3}(\mathrm{mb})$ & $\begin{array}{l}\log y_{3} \\
(S i=6)\end{array}$ & $\mathrm{n}_{\mathrm{n}}\left(\mathrm{cm}^{-3}\right)$ & $\lg n_{n}$ \\
\hline 39. & $114 \mathrm{Cd}$ & $-0,333$ & 135,3 & 44,56 & $\mathrm{~d}^{*}$ & $115 \mathrm{Cd}^{\mathrm{m}}$ & 224 & $115 \mathrm{In}$ & 776 & $-0,754$ & $-1,60 \mathrm{E}+09$ & $\mathrm{n} / \mathrm{a}$ \\
\hline 40. & $113 \ln$ & $-2,103$ & 229 & 71,9 & $\mathrm{~s}$ & 114In & $\underline{1308}$ & $114 S n$ & 134,4 & $-1,599$ & $-1,26 E+13$ & $n / a$ \\
\hline 41. & $113 \ln$ & $-2,103$ & 580 & 49,51 & $\mathrm{~d}^{*}$ & $114 \ln ^{\mathrm{m}}$ & 2595 & $114 \mathrm{Sn}$ & 134,4 & $-1,599$ & $8,07 \mathrm{E}+07$ & 7,91 \\
\hline 42. & $115 \ln$ & $-0,754$ & 154 & 14,1 & $\mathrm{~s}$ & $116 \mathrm{In}$ & 1377 & $116 \mathrm{Sn}$ & 92,3 & $-0,263$ & $-5,91 E+13$ & $\mathrm{n} / \mathrm{a}$ \\
\hline 43. & $115 \ln$ & $-0,754$ & 622 & 54,29 & $\mathrm{~m}$ & $116 \mathrm{In}^{\mathrm{m}}$ & 1377 & $116 \mathrm{Sn}$ & 92,3 & $-0,263$ & $6,51 \mathrm{E}+11$ & 11,81 \\
\hline 44. & $120 S n$ & 0,097 & 36,3 & 27,03 & $\mathrm{~h}$ & $121 \mathrm{Sn}$ & 167 & $121 \mathrm{Sb}$ & 532 & $-0,752$ & $-2,40 E+10$ & $n / a$ \\
\hline 45. & $120 \mathrm{Sn}$ & 0,097 & 36,3 & 43,9 & $y^{*}$ & $121 \mathrm{Sn}^{\mathrm{m}}$ & 175,4 & $121 \mathrm{Sb}$ & 532 & $-0,752$ & $-1,60 \mathrm{E}+06$ & $\mathrm{n} / \mathrm{a}$ \\
\hline 46. & $122 \mathrm{Sn}$ & $-0,745$ & 22,6 & 129,2 & $d$ & $123 S n$ & 361 & $123 \mathrm{Sb}$ & 303 & $-0,879$ & $-3,00 E+11$ & $n / a$ \\
\hline 47. & $122 \mathrm{Sn}$ & $-0,745$ & 21,7 & 40,06 & $\mathrm{~m}$ & $123 \mathrm{Sn}^{\mathrm{m}}$ & 361 & $123 \mathrm{Sb}$ & 303 & $-0,879$ & $-1,40 \mathrm{E}+15$ & $\mathrm{n} / \mathrm{a}$ \\
\hline 48. & $121 \mathrm{Sb}$ & $-0,752$ & 532 & 2,7238 & $d$ & $122 \mathrm{Sb}$ & 894 & $122 \mathrm{Te}$ & 295,4 & $-0,928$ & $7,76 \mathrm{E}+10$ & 10,89 \\
\hline 49. & $123 \mathrm{Sb}$ & $-0,879$ & 303 & 60,11 & $d$ & $124 \mathrm{Sb}$ & 924,0 & $124 \mathrm{Te}$ & 155 & $-0,654$ & $5,00 \mathrm{E}+11$ & 11,70 \\
\hline 50. & $126 \mathrm{Te}$ & $-0,046$ & 81,3 & 9,35 & $\mathrm{~h}$ & $127 \mathrm{Te}$ & 256,8 & $127 \mid$ & 662 & $-0,046$ & $-2,23 E+11$ & $\mathrm{n} / \mathrm{a}$ \\
\hline 51. & $126 \mathrm{Te}$ & $-0,046$ & 81,3 & 109 & $d^{*}$ & $127 \mathrm{Te}^{\mathrm{m}}$ & 668,6 & $127 \mid$ & 662 & $-0,046$ & $-3,06 E+08$ & $\mathrm{n} / \mathrm{a}$ \\
\hline 52. & $127 \mid$ & $-0,046$ & 662 & 24,99 & $\mathrm{~m}$ & $128 I$ & 679,5 & $128 X e$ & 262,5 & 0,991 & $-1,83 \mathrm{E}+12$ & $\mathrm{n} / \mathrm{a}$ \\
\hline 53. & $132 X e$ & 0,086 & 63,8 & 5,243 & d & $133 \mathrm{Xe}$ & 127 & $133 \mathrm{Cs}$ & 502 & $-0,429$ & $-3,64 E+10$ & $n / a$ \\
\hline 54. & $133 \mathrm{Cs}$ & $-0,429$ & 502 & 2,0652 & $y$ & $134 \mathrm{Cs}$ & 724 & 134Ba & 176 & $-0,963$ & $9,52 \mathrm{E}+10$ & 10,98 \\
\hline 55. & $138 \mathrm{Ba}$ & 0,508 & 4,13 & 83,06 & $\mathrm{~m}$ & 139Ba & 39,7 & 139La & 32,4 & $-0,351$ & $-1,02 \mathrm{E}+12$ & $\mathrm{n} / \mathrm{a}$ \\
\hline 56. & 139La & $-0,351$ & 32,4 & 1,67855 & d & 140La & 117,8 & $140 \mathrm{Ce}$ & 11,73 & 0,004 & $4,11 \mathrm{E}+10$ & 10,61 \\
\hline 57. & $140 \mathrm{Ce}$ & 0,004 & 11,73 & 32,508 & $d$ & $141 \mathrm{Ce}$ & 76 & $141 \mathrm{Pr}$ & 111,4 & $-0,777$ & $-4,35 E+09$ & $n / a$ \\
\hline 58. & $141 \mathrm{Pr}$ & $-0,777$ & 111,4 & 19,12 & $\mathrm{~h}$ & $142 \mathrm{Pr}$ & 361,4 & $142 \mathrm{Nd}$ & 35,1 & $-0,65$ & $3,60 \mathrm{E}+10$ & 10,56 \\
\hline 59. & $150 \mathrm{Sm}$ & $-1,717$ & 422,3 & 90 & $y$ & $151 \mathrm{Sm}$ & 3040 & $151 \mathrm{Eu}$ & 3556 & 0,35 & $-7,31 \mathrm{E}+06$ & $\mathrm{n} / \mathrm{a}$ \\
\hline 60. & $152 \mathrm{Sm}$ & $-1,161$ & 464,8 & 46,284 & $\mathrm{~h}$ & $153 \mathrm{Sm}$ & 1095 & 153Eu & 2567 & $-1,294$ & $-1,81 \mathrm{E}+10$ & $\mathrm{n} / \mathrm{a}$ \\
\hline 61. & 153Eu & $-1,294$ & 2557 & 8,59 & $y$ & $154 \mathrm{Eu}$ & 4420 & $154 \mathrm{Gd}$ & 1028 & $-2,149$ & $1,08 \mathrm{E}+10$ & 10,03 \\
\hline 62. & $158 \mathrm{Gd}$ & $-1,086$ & 323,6 & 18,479 & $\mathrm{~h}$ & $159 \mathrm{Gd}$ & 455,2 & 159Tb & 1817 & $-1,22$ & $-6,09 E+10$ & $\mathrm{n} / \mathrm{a}$ \\
\hline 63. & 159Tb & $-1,22$ & 1817 & 72,3 & $d$ & $160 \mathrm{~Tb}$ & 3240 & 160Dy & 890 & $-2,044$ & $2,52 \mathrm{E}+11$ & 11,40 \\
\hline 64. & 164Dy & $-0,955$ & 212 & 2,334 & $\mathrm{~h}$ & 165Dy & 284,5 & $165 \mathrm{Ho}$ & 1237 & $-1,051$ & $-8,43 E+11$ & $\mathrm{n} / \mathrm{a}$ \\
\hline 65. & $165 \mathrm{Ho}$ & $-1,051$ & 1237 & 26,83 & $\mathrm{~h}$ & $166 \mathrm{Ho}$ & 1262,0 & $166 \mathrm{Er}$ & 700 & $-1,077$ & $1,16 \mathrm{E}+10$ & 10,07 \\
\hline 66. & $165 \mathrm{Ho}$ & $-1,051$ & 1237 & $1,20 E+03$ & $y$ & $166 \mathrm{Ho}^{\mathrm{m}}$ & 1235,3 & $166 \mathrm{Er}$ & 700 & $-1,077$ & $3,03 E+04$ & 4,48 \\
\hline 67. & $168 \mathrm{Er}$ & $-1,168$ & 319 & 9,392 & d & $169 \mathrm{Er}$ & 653 & 169Tm & 1065 & $-1,423$ & $-2,40 \mathrm{E}+09$ & $\mathrm{n} / \mathrm{a}$ \\
\hline 68. & $169 \mathrm{Tm}$ & $-1,423$ & 1065 & 128,6 & $d$ & $170 \mathrm{Tm}$ & 1870 & $170 \mathrm{Yb}$ & 768,3 & $-2,124$ & $7,95 E+08$ & 8,90 \\
\hline 69. & $174 \mathrm{Yb}$ & $-1,103$ & 150,5 & 4,158 & d & $175 \mathrm{Yb}$ & 558 & $175 \mathrm{Lu}$ & 1219 & $-1,449$ & $-1,64 \mathrm{E}+10$ & $\mathrm{n} / \mathrm{a}$ \\
\hline 70. & 176Lu & $-2,959$ & 1639 & 6,6475 & d & 177Lu & 794,9 & $177 \mathrm{Hf}$ & 1544 & $-1,545$ & $-8,18 E+09$ & $\mathrm{n} / \mathrm{a}$ \\
\hline 71. & 176Lu & $-2,959$ & 1639 & 160,44 & $d$ & $177 \mathrm{Lu}^{\mathrm{m}}$ & 348,3 & $177 \mathrm{Hf}$ & 1544 & $-1,545$ & $-7,73 E+08$ & $\mathrm{n} / \mathrm{a}$ \\
\hline 72. & 176Lu & $-2,959$ & 1639 & 6 & $\mathrm{~m}$ & $177 \mathrm{Lu}^{\mathrm{m}}$ & 348,3 & $177 \mathrm{Hf}$ & 1544 & $-1,545$ & $-2,98 E+13$ & $\mathrm{n} / \mathrm{a}$ \\
\hline 73. & $180 \mathrm{Hf}$ & $-1,265$ & 156,6 & 42,39 & $d$ & $181 \mathrm{Hf}$ & 194 & $181 \mathrm{Ta}$ & 766 & $-1,684$ & $-5,63 E+10$ & $\mathrm{n} / \mathrm{a}$ \\
\hline 74. & $181 \mathrm{Ta}$ & $-1,684$ & 766 & 114,43 & $d$ & $182 \mathrm{Ta}$ & 1120 & $182 W$ & 285 & $-1,455$ & $5,21 \mathrm{E}+08$ & 8,72 \\
\hline 75. & $184 \mathrm{~W}$ & $-1,389$ & 225 & 75,4 & $d$ & $185 W$ & 633 & $185 \operatorname{Re}$ & 1438,5 & $-1,717$ & $-4,83 E+08$ & $\mathrm{n} / \mathrm{a}$ \\
\hline
\end{tabular}




\begin{tabular}{|c|c|c|c|c|c|c|c|c|c|c|c|c|}
\hline & nuc $_{1}$ & $\begin{array}{l}\log y_{1} \\
(S i=6)\end{array}$ & $\sigma_{1}(\mathrm{mb}$ & $T_{2}$ & $\begin{array}{l}\text { time } \\
\text { unit }\end{array}$ & nuc $_{2}$ & $\sigma_{2}(m b)$ & nuc $_{3}$ & $\sigma_{3}(\mathrm{mb})$ & $\begin{array}{l}\log y_{3} \\
(S i=6)\end{array}$ & $\mathrm{n}_{\mathrm{n}}\left(\mathrm{cm}^{-3}\right)$ & $\lg n_{n}$ \\
\hline 76. & $186 W$ & $-1,423$ & 226 & 23,72 & $\mathrm{~h}$ & $187 \mathrm{~W}$ & $\underline{183}$ & $187 \mathrm{Re}$ & 1184 & $-1,475$ & $-1,41 E+11$ & $\mathrm{n} / \mathrm{a}$ \\
\hline 77. & $185 \mathrm{Re}$ & $-1,717$ & 1420 & 3,7186 & $d$ & $186 \mathrm{Re}$ & 743 & $1860 s$ & 414 & $-2,06$ & $7,48 \mathrm{E}+10$ & 10,87 \\
\hline 78. & $185 \mathrm{Re}$ & $-1,717$ & 18,5 & $2,00 E+05$ & $\mathrm{y}^{*}$ & $186 \mathrm{Re}^{\mathrm{m}}$ & 743 & $1860 s$ & 414 & $-2,06$ & $-5,23 E+02$ & $\mathrm{n} / \mathrm{a}$ \\
\hline 79. & $187 \mathrm{Re}$ & $-1,475$ & 1187 & 17,003 & $\mathrm{~h}$ & $188 \mathrm{Re}$ & 2343 & $1880 s$ & 294 & $-1,047$ & $9,50 \mathrm{E}+09$ & 9,98 \\
\hline 80. & $1900 \mathrm{~s}$ & $-0,75$ & 278 & 15,4 & $d$ & 1910s & 1290 & $191 / r$ & 1350 & $-0,607$ & $-2,58 E+09$ & $\mathrm{n} / \mathrm{a}$ \\
\hline 81. & $1920 s$ & $-0,558$ & 160 & 30,11 & $\mathrm{~h}$ & 1930 s & 95,6 & $193 / r$ & 994 & $-0,383$ & $-2,05 E+11$ & $\mathrm{n} / \mathrm{a}$ \\
\hline 82. & $191 / \mathrm{l}$ & $-0,607$ & 1350 & $7,38 \mathrm{E}+01$ & $d$ & $192 \mathrm{Ir}$ & 2080 & $192 \mathrm{Pt}$ & 483 & $-1,979$ & $1,76 \mathrm{E}+10$ & 10,24 \\
\hline 83. & $191 / \mathrm{l}$ & $-0,607$ & 1350 & $2,41 \mathrm{E}+02$ & $y^{*}$ & $192 \mathrm{Ir}^{\mathrm{m}}$ & 2080 & $192 \mathrm{Pt}$ & 483 & $-1,979$ & $1,47 \mathrm{E}+07$ & 7,17 \\
\hline 84. & 193Ir & $-0,383$ & 994 & 19,28 & $\mathrm{~h}$ & $194 \mathrm{Ir}$ & 397,8 & $194 \mathrm{Pt}$ & 283 & $-0,356$ & $3,47 \mathrm{E}+11$ & 11,54 \\
\hline 85. & $196 \mathrm{Pt}$ & $-0,47$ & 167,4 & 19,8915 & $\mathrm{~h}$ & $197 \mathrm{Pt}$ & 175,4 & 197Au & 612,8 & $-0,728$ & $-1,19 E+11$ & $\mathrm{n} / \mathrm{a}$ \\
\hline 86. & 197Au & $-0,728$ & 612,8 & $2,70 \mathrm{E}+00$ & $d$ & 198Au & 840 & $198 \mathrm{Hg}$ & 173 & $-1,46$ & $9,52 \mathrm{E}+11$ & 11,98 \\
\hline 87. & $202 \mathrm{Hg}$ & $-0,996$ & 63,3 & 46,594 & $d$ & $203 \mathrm{Hg}$ & 98 & $203 \mathrm{TI}$ & 170,5 & $-1,265$ & $-1,40 \mathrm{E}+11$ & $\mathrm{n} / \mathrm{a}$ \\
\hline 88. & $204 \mathrm{Hg}$ & $-1,633$ & 42 & 5,14 & $\mathrm{~m}$ & $205 \mathrm{Hg}$ & $\underline{12,7}$ & $205 \mathrm{TI}$ & 52,6 & $-0,886$ & $-5,59 E+14$ & $\mathrm{n} / \mathrm{a}$ \\
\hline 89. & 203TI & $-1,265$ & 170,5 & 3,78 & $y$ & $204 \mathrm{TI}$ & 215 & $204 \mathrm{~Pb}$ & 83,7 & $-1,207$ & $1,55 \mathrm{E}+10$ & 10,19 \\
\hline 90. & $205 \mathrm{TI}$ & $-0,866$ & 52,6 & 4,2 & $\mathrm{~m}$ & $206 \mathrm{TI}$ & 34,1 & $206 \mathrm{~Pb}$ & 14,7 & $-0,277$ & $-2,32 \mathrm{E}+13$ & $\mathrm{n} / \mathrm{a}$ \\
\hline 91. & $208 \mathrm{~Pb}$ & 0,265 & 0,376 & 3,253 & $\mathrm{~h}$ & $209 \mathrm{~Pb}$ & 3,6 & $209 \mathrm{Bi}$ & 2,61 & $-0,842$ & $5,10 \mathrm{E}+13$ & 13,71 \\
\hline
\end{tabular}

\section{Legend}

n/a: $\quad$ not all third nuclei can be achieved in that process

$\log \mathrm{y}_{\mathrm{i}}: \quad$ D. Arnett: Supernovae and Nucleosynthesis, Princeton University Press, 1996 at $\sigma_{i}$ :

bold: $\quad$ KADoNiS 1.0

normal: $\quad$ KADoNiS 0.3 (MACS)

italic: $\quad$ B. Pritychenko,* S.F. Mughabghab: Neutron Thermal Cross Sections, Westcott Factors, Resonance Integrals, Maxwellian Averaged Cross Sections and Astrophysical Reaction Rates Calculated from the ENDF/B-VII.1, JEFF-3.1.2, JENDL-4.0, ROSFOND2010, CENDL-3.1 and EAF-2010 Evaluated Data Libraries underlined: http://adg.llnl.gov/Research/RRSN/aemr/30kev/rath00_7.4.30kev_calc 